RUNNING HEAD: PROBABILITY RANGE AND DISTORTION

\title{
Probability range and probability distortion in a gambling task
}

\author{
C. Xing, ${ }^{1}$ J. Paul, ${ }^{1}$ A. Zax, ${ }^{1}$ S. Cordes,${ }^{2}$ H. Barth, ${ }^{1}$ \& A. L. Patalano ${ }^{1}$ \\ ${ }^{1}$ Department of Psychology, Wesleyan University \\ ${ }^{2}$ Department of Psychology, Boston College
}

Accepted for publication, Acta Psychologica

This copy: Preprint 1.0 (March 2019)

Word count: $\sim 13,468$ including abstract, table and figure descriptions, and references Date: March 2, 2019

Address correspondence to: Andrea Patalano, Department of Psychology, Wesleyan University, 207 High St., Middletown CT 06459, USA, 860-685-2310, apatalano@wesleyan.edu

Author note: We thank lab coordinator Katie Williams, and research assistants Marcia Mata, Liana Mathias, and Lily Segal. This work was supported by funding from NSF DRL-1561214 and Wesleyan University.

Declaration of interest: National Science Foundation grant (NSF DRL-1561214). Other than funding support, NSF played no role in conducting the research or writing the report. 


\begin{abstract}
In decision making under risk, adults tend to overestimate small and underestimate large probabilities (Tversky \& Kahneman, 1992). This inverse S-shaped distortion pattern is similar to that observed in a wide variety of proportion judgment tasks (see Hollands \& Dyre, 2000, for review). In proportion judgment tasks, distortion patterns tend not to be fixed but rather to depend on the reference points to which the targets are compared. Here, we tested the novel hypothesis that probability distortion in decision making under risk might also be influenced by reference points - in this case, references implied by the probability range. Adult participants were assigned to either a full-range (probabilities from 0-100\%), upper-range (50-100\%), or lower-range $(0-50 \%)$ condition, where they indicated certainty equivalents for 176 hypothetical monetary gambles (e.g., "a 50\% chance of $\$ 100$, otherwise $\$ 0$ "). Using a modified cumulative prospect theory model, we found only minimal differences in probability distortion as a function of condition, suggesting no differences in use of reference points by condition, and broadly demonstrating the robustness of distortion pattern across contexts. However, we also observed deviations from the curve across all conditions that warrant further research.
\end{abstract}

Keywords: Probability; proportion judgment; cumulative prospect theory; decision making under risk; numeracy 


\section{Probability range and probability distortion in a gambling task}

\section{Introduction}

\subsection{Cumulative prospect theory framework}

Imagine a gamble involving a 50\% chance of winning \$100, otherwise \$0. One’s certainty equivalent $(C E)$ for such a gamble is defined as the monetary outcome identified by the individual as being as attractive as playing the gamble, thus reflecting the overall value of the gamble to the individual. According to cumulative prospect theory (CPT), a dominant descriptive theory of decision-making under risk (Tversky \& Kahneman, 1992; see Glöckner \& Pachur, 2012, for comparison to other models), CEs for simple gambles with two possible nonnegative outcomes represented as $\left(x_{1}, p ; x_{2}, 1-p\right)$, with probabilities expressed as decimal values from 0 to 1 can be modeled as:

$$
\begin{gathered}
v(C E)=v\left(x_{1}\right) w(p)+v\left(x_{2}\right)(1-w(p))^{1} \\
\text { where } x_{1}>x_{2} \geq 0 \text { and } 0 \leq p \leq 1
\end{gathered}
$$

One component of CPT reflected in the equation is that outcomes (including the certainty equivalent) and probabilities are transformed into subjective values and decision weights respectively before they are combined. A second component of CPT is that the subjective value of the $C E$ is essentially the sum of the subjective value of each gamble outcome multiplied by its corresponding decision weight. The transformation of outcomes into subjective values is assumed to follow a concave curve for gains (reflecting diminishing sensitivity as outcome magnitude increases) and a convex curve for losses, with the curve being steeper for losses than for gains (see Fig. 1a for the curve associated with gains). The transformation of probabilities

\footnotetext{
${ }^{1}$ Probabilities are represented using decimal format (0-1 range) in this CPT equation, per convention and ease of use, but as percentages (0-100\% range) elsewhere here, including in the gambles presented to participants in our studies.
} 
into decision weights is assumed to follow an inverse S-shaped curve, reflecting an overweighting of small probabilities (e.g., behaving as if a $10 \%$ chance is more likely than it actually is) and an underweighting of medium to large ones (see Fig. 1b). These general forms of distortion curves have been used to explain a complex pattern of behavior: risk aversion for high probability gains and risk seeking for high probability losses, but the reverse for low probability gains and losses (Tversky \& Kahneman, 1992).

With regard to specific value and probability weighting functions, the CPT value function (we focus on the function for gains; an additional parameter is needed to accommodate losses) is typically specified as (Tversky \& Kahneman, 1992; see Fox \& Poldrack, 2014, for review):

$$
v(x)=x^{\alpha},
$$

where $\alpha>0$.

The value parameter $\alpha$ captures degree of curvature from the identity line: $\alpha=1$ is the identity relationship, $\alpha<1$ is a concave curve (as shown in Fig. 1a), and $\alpha>1$ is a convex curve. For probability weighting, several different specifications of the inverse S-shaped curve have been proposed (for reviews, see Fox \& Poldrack, 2014; Stott, 2006), including the one-parameter function originally proposed by Tversky and Kahneman (1992):

$$
\begin{gathered}
w(p)=\left(p^{\gamma} /\left(p^{\gamma}+(1-p)^{\gamma}\right)\right)^{1 / \gamma}, \\
\text { where } \gamma>0 .
\end{gathered}
$$

With the one-parameter function, the single parameter $\gamma$ determines both degree of curvature and where the curve crosses the identity line (i.e., curvature and crossover are not independent). An alternative function with two parameters is one of the most commonly used (see Fox \& Poldrack, 2014) because its parameters have a more natural interpretation. It has been shown to generally provide a better fit to individual-level data (Cavagnaro, Pitt, Gonzalez, \& Myung, 2013). This 
alternative is Lattimore, Baker, and Witte's (1992; see also Goldstein \& Einhorn, 1987;

Gonzalez \& Wu, 1999) function:

$$
\begin{gathered}
w(p)=\delta p^{\gamma} /\left(\delta p^{\gamma}+(1-p)^{\gamma}\right), \\
\text { where } \delta>0 \text { and } \gamma>0 .
\end{gathered}
$$

The parameter $\gamma$ captures degree of curvature from the identity line: $\gamma=1$ is the identity relationship, $\gamma<1$ is an inverse S-shaped curve (as shown in Fig. 1c), and $\gamma>1$ is an S-shaped curve. The parameter $\delta$ captures elevation of the entire curve, which largely determines where it crosses the identity line: $\delta=1$ crosses at a probability of .5 , while $\delta>1$ crosses above .5 and $\delta<$ 1 crosses below .5 (examples shown in Fig. 1d). While CPT as proposed by Tversky and Kahneman (1992) includes among its key elements that the value curve is concave for gains and that the probability weighting curve is inverse S-shaped, the parameters of the CPT equation are not formally constrained to being below 1, and individual-level estimates are often above 1 (e.g., Patalano et al., 2015; Gonzalez \& Wu, 1999). As with past decision making work, we generally focus on the inverse S-shaped pattern here, but all equations discussed can produce S-shaped curves as well, and thus can be used to describe this less common pattern too.

\section{FIGURE 1 ABOUT HERE}

A number of psychological explanations for the inverse-S shape of the probability weighting curve have been proposed. For example, Tversky and Kahneman (1992) proposed the notion of diminishing sensitivity to probabilities as a function of their distance from reference points of impossibility and certainty (namely 0 and 100\%). According to venture theory, proposed by Hogarth and Einhorn (1990), one anchors on a stated probability and then adjusts by mentally simulating other possible values. The adjustment is generally expected to be in the direction in which there are more possible values, thereby giving rise to over- and 
underestimation patterns. Rottenstreich and Hsee (2001; but see Klein et al., 2018) developed an affect-based account of probability weighting by which hope drives overestimation of low probabilities and fear drives underestimation of large probabilities. Though Tversky and Kahneman's account is inspired by psychophysics (Kahneman \& Tversky, 1984), all of the explanations described thus far generally assume that the pattern of bias arises from inputs and mental processes specific to decision making, rather than from broader characteristics of cognition. These explanations also generally assume that the mapping of probability magnitudes to decision weights is fixed (e.g., not dependent on context) and that probability weighting can be accommodated by a single psychological explanation. To our knowledge, only Gonzalez and $\mathrm{Wu}$ (1999) proposed distinct psychological sources of probability weighting curvature and elevation, namely, that curvature is associated with discrimination of probabilities (greater curvature indicates less discrimination), while elevation is associated with the overall attractiveness of gambling to the individual (greater elevation indicates greater attractiveness). Finally, in Stewart, Chater, and Brown's (2006; Stewart, 2009; Stewart, Reimers, \& Harris, 2015) decision by sampling theory — an approach that does not assume a fixed mapping - the probability weighting curve is thought to emerge dynamically in some contexts as a result of evaluating probabilities by ranking them relative to other probabilities sampled from the decision environment (but see Alempaki, et al., in press, for alternative account).

\subsection{Proportion judgment approach}

Though not frequently discussed in the decision literature, similar distortion patterns have been found in a wide variety of cognitive, perceptual, and motor tasks varying in task demands and types of stimuli (for reviews, see Hollands \& Dyre, 2000; Zhang \& Maloney, 2012). What these tasks have in common is that they can be conceptualized as involving proportion judgment. 
For example, in a task involving judging the proportion of black dots in a visual display of black and white dots, individuals tend to overestimate small proportions of black dots (e.g., saying .30 or $30 \%$ when the correct response is .20 or $20 \%$ ) and to underestimate large ones, showing a symmetrical (that is, with a crossover of the identity line at .50) inverse S-shaped pattern (Varey, Mellers, \& Birnbaum, 1990). Similarly, in a number line task involving the placement of numbers on a line labeled only at its endpoints (e.g., with 0 and 100), participants tend to overestimate the position of small numbers and underestimate the position of large ones, again showing the symmetrical inverse S-shaped pattern (e.g., Cohen \& Blanc-Goldhammer, 2011; Slusser \& Barth, 2017; Sullivan, Juhasz, Slattery, \& Barth, 2011). Although less frequently, asymmetrical patterns of bias (e.g., an inverse S-shaped curve with a crossover at .30) have also been observed (e.g., Spence, 1990). These findings have raised the possibility of common psychological explanations across domains in which the task can be conceptualized as one of proportion judgment.

Hollands and Dyre (2000) developed a model in the psychophysical tradition, called the cyclical power model (CPM), that accounts for estimation biases resulting from a wide variety of perceptually based tasks. It has recently been extended to abstract symbolic representations of quantities such as the number line task (Barth \& Paladino, 2011; Cohen \& Blanc-Goldhammer, 2011; Sullivan et al., 2011). In the CPM, bias in proportion estimation is thought to originate from bias in the estimation of each part's magnitude, in that "psychological magnitude" is not the same as true physical magnitude. The CPM builds on Stevens' Law (Stevens, 1957) which describes the relationship between the estimated or perceived magnitude of a stimulus and its actual magnitude. Steven's Law is a power function:

$$
y=\delta x^{\gamma}
$$




$$
\text { where } x>0, \delta>0 \text {, and } \gamma>0 \text {, }
$$

where $\gamma$ quantifies bias associated with judgments of a particular stimulus continuum (e.g. brightness or area) and $\delta$ is a scaling parameter. Spence (1990) extended Steven's power law (Eq. 5) to proportion judgments, demonstrating that one's judgment of the proportion $(P)$ of a part to a whole (e.g., the relative area of one square to the whole of two squares) can be expressed as:

$$
\begin{gathered}
P(x)=x^{\gamma} /\left(x^{\gamma}+(1-x)^{\gamma}\right) \\
\text { where } 0 \leq x \leq 1,
\end{gathered}
$$

where 1 reflects the whole, $x$ reflects the true proportion of the stimulus to the whole, and $\gamma$ refers to the same value used in Stevens' Law (Eq. 5). Spence's power model produces a symmetrical inverse S-shaped curve when $\gamma<1$ and an S-shaped curve when $\gamma>1$.

Hollands and Dyre's (2000) CPM is a modified and expanded version of Spence's power model (Eq. 6) in which stimuli can be expressed in any unit of magnitude (e.g., 20 out of a whole of 80 , or 4 out of a whole of 16 , rather than only as the proportion .25 out of a whole of 1 ), and in which reference points (which can vary by individual and context) can be used to constrain the range over which estimates of magnitude are made. Hollands and Dyre (2000) proposed that when judging a proportion, an individual might use two bounding reference points, resulting in the symmetrical one-cycle pattern of bias (as in Fig. 2). Such a pattern might arise, for example, in a number line task if one judged the location of ' 20 ' on a line bounded by the labels 0 to 100 , using only these two bounding reference points. Hollands and Dyre further proposed that additional intermediate reference points might also be used, leading to multi-cycle bias patterns, such as the symmetrical two-cycle pattern in Figure 2 (which assumes one additional middle reference point). Such a pattern would arise in the number line task if one used the middle of the 
line as an additional reference point. In this case, ' 20 ' would be estimated with reference to the range between 0 and 50 , while a number such as ' 65 ' would be estimated with reference to the range between 50 and 100. Two-cycle bias patterns have been observed in diverse perceptual judgments (see Hollands \& Dyre, 2000) and also in children's number-line estimates, with developmental change arising in part from the increasing use of additional reference points with age (Barth \& Paladino, 2011; Cohen \& Sarnecka, 2014; Link, Huber, Nuerk, \& Moeller, 2014; Rouder \& Geary, 2014; see Slusser, Santiago, \& Barth, 2013, for details). The CPM can explain a wide range of symmetrical (and some asymmetrical) multi-cycle patterns through the mechanism of reference points.

\section{FIGURE 2 ABOUT HERE}

Hollands and Dyre developed a general equation that can accommodate any defined number of reference points (though we focus on one-cycle and two-cycle patterns here, with two and three reference points respectively). They also developed a mixed model to accommodate situations in which one set of reference points is used for one subset of responses and a different set of reference points is used for another subset of responses (as in Fig. 2 which illustrates a combination of a one-cycle and a two-cycle pattern). According to the CPM, when observers respond using two or more reference points in their judgments of the relationship of a part to a whole, proportion estimates are predicted by:

$$
\begin{gathered}
P(x)=\left(\left(x-R_{i-1}\right)^{\gamma} /\left(\left(x-R_{i-1}\right)^{\gamma}+\left(R_{i}-x\right)^{\gamma}\right)\right) \cdot\left(R_{i}-R_{i-1}\right) /\left(\mathrm{R}_{n}-\mathrm{R}_{0}\right)+\mathrm{R}_{i-1} / \mathrm{R}_{n} \\
\text { where } R_{\mathrm{i}-1} \leq x \leq R_{i},
\end{gathered}
$$

where $x$ is the stimulus magnitude, $R$ is a vector of reference values from $R_{0}$ to $R_{n}$ (where $R_{0}$ and $R_{n}$ are the boundary values for the entire range), $R_{i-1}$ is the lowerbound and $R_{i}$ is the upperbound of the immediate range containing $x$, and $P(x)$ is the estimated proportion that $x$ represents of the 
whole. If there are only two reference points (a one-cycle pattern), the first part of the expression (until the multiplication sign) returns a proportion between 0 and 1 , and the rest of the equation reduces to identity operations. However, if there are three equally-spaced reference values (a two-cycle pattern), the first part of the equation returns a proportion between 0 and 1 (e.g., .8), the expression $\left(R_{i}-R_{i-1}\right) /\left(R_{n}-R_{0}\right)$ (which always equals .5 in the case of three equallyspaced reference points) scales the proportion to the reference range (e.g., $.5 * .8=.4$ ), and the expression $\mathrm{R}_{i-1} / \mathrm{R}_{n}$ adds the proportion to the lower boundary of the reference range (which is either 0 or .5 in the case of three equally-spaced reference points). Further, a mixed model that incorporates the use of two reference points for some judgments and three for others can be written simply as:

$$
\begin{gathered}
P(x)=\omega(\text { one-cycle equation })+(1-\omega) \text { (two-cycle equation), } \\
\text { where } 0 \leq \omega \leq 1,
\end{gathered}
$$

where the first equation assumes two reference points and the second assumes three. The $\omega$ is an estimated parameter that reflects the weight assigned to each pattern in the best-fitting model (where $\omega=1$ indicates a pure one-cycle pattern, and $\omega=0$ indicates a pure two-cycle pattern). Noteworthy about the mixed model is that it predicts bias patterns that are not necessarily symmetrical (because a weighted sum of two symmetrical patterns is not always symmetrical) and thereby is able to explain some asymmetrical patterns without need for any additional parameter (other than the weighting parameter) or psychological account of the crossover.

\subsection{Integrating proportion judgment approach into decision models}

It should now be apparent that probability distortion patterns in decision making are similar to those seen in other domains for which a proportion judgment account has been proposed and that the equations that have been fit to data are also very similar across domains. 
In particular, the probability weighting functions introduced earlier (Eqs. 3 and 4) are both identical to Spence's power model equation (Eq. 6) with the exception that each of the probability weighting functions includes a means of producing the asymmetrical distortion pattern (e.g., by raising or lowering the curve; see Fig. 1c) often seen in decision making. ${ }^{2}$ While proportion judgment does not provide an obvious explanation for the elevation of the probability weighting curve (which may have a distinct psychological source, such as the attractiveness of gambling to the individual; see Gonzalez \& Wu, 1999) — it does offer a straightfoward account of the shape of the curve. We are interested in the intriguing question of whether adopting a proportion judgment approach might lead to new insights regarding probability distortion in decision making, in particular regarding the shape of the probability weighting curve.

According to Spence's power model (Eq. 6), distortion in proportion judgment arises from imprecision in the estimation of magnitudes contributing to the judgment. Thus one insight from a proportion judgment perspective, although not the focus of the present work, is that there might be a link between our intuitive magnitude system (see Libertus \& Brannon, 2009, for review) and distortion in the use of numbers in decision making. Researchers have begun to study the link between intuitive magnitude skills (e.g., judging numerosity of dots in a display) and use of numbers in decision making (e.g., Patalano, Saltiel, Machlin, \& Barth, 2015; Peters, Slovic, Västfjäll, \& Mertz, 2008; Schley \& Peters, 2014; see also Reyna, Nelson, Han, \& Dieckmann, 2009; Winman, Juslin, Lindskog, Nilsson, \& Kerimi, 2014), providing initial evidence that several intuitive magnitude estimation measures are related to value and probability distortion. Numeracy measures tapping into fluency in processing part-whole relationships (e.g., difficulty translating from fractions to percentages) have also been associated

\footnotetext{
${ }^{2}$ One earlier quantitative description of probability distortion in decision-making (Karmarkar, 1978) is formally identical to the Spence (1990) proportion judgment model, though its psychological derivation was unrelated.
} 
with value and probability distortion and with overall CPT model fit and response reliability (e.g., Patalano et al., 2015; Schley \& Peters, 2014). These findings provide initial evidence that value and probability distortion in decision making might arise not (only) from psychological sources specific to decision making but, rather, from a more general property of an intuitive magnitude system involved in a wide range of magnitude and proportion-related judgments, evidence consistent with a proportion judgment account.

The focus of the present work is a prediction arising specifically from application of the cyclical power model to the distortion of probabilities in decision making. According to the CPM, whether a value is over- or underestimated depends not on its absolute magnitude but rather on the proportional relationship between it and the reference range to which it is compared. The type of cyclical pattern of bias that emerges in any context (e.g., one-cycle vs. two-cycle patterns) depends on the number of reference points used. In decision making, probability weighting curves are assumed to follow a one-cycle pattern, with an inverse S-shaped curve extending across the full range of values. A novel prediction that follows if the CPM is applied to decision making is that contexts that facilitate the use of additional reference points during the evaluation of probabilities should give rise to multi-cycle patterns of probability distortion. For example, while a one-cycle pattern might emerge when only 0 and 100 are used as reference points in probability weighting (Fig. 3a), a two-cycle pattern might emerge when 50 is available as an additional reference point (Fig. 3b). Instead, if reference values do not constrain proportion judgment in decision making contexts, or if proportion judgment does not underlie the interpretation of probabilities in decision making, then efforts to influence reference points should have little effect on distortion patterns. This specific prediction has not yet been tested, although there is considerable evidence that how a probability is evaluated fluctuates in 
response to one's recent experience with other probabilities in the environment (e.g., Ungemach, Stewart, \& Reimers, 2011; Wedell, 1991; Windschitl \& Chambers, 2004).

\section{FIGURE 3 ABOUT HERE}

\subsection{Developing a cyclical CPT equation}

Towards testing whether a one- or two-cycle probability weighting pattern might better fit $C E$ data, we developed a modified probability weighting function by incorporating elements of the CPM into an existing probability weighting function. We took Lattimore et al.'s (1992) two-parameter probability weighting function (Eq. 4) as our starting point. We used this function rather than, for example, Tversky and Kahenman's (1992) one-parameter function because the Lattimore et al. function is more commonly used in the field (see Fox \& Poldrack, 2014), it better fits individual-level data and, importantly, it reflects our perspective that probability weighting curvature and elevation may have different psychological sources. From this starting point, the equation was generalized to accept input of any unit of magnitude (e.g., 80 out of 100 rather than only .8 out of 1) for consistency with the CPM (Eq. 7; although this choice does not affect model predictions), and we introduced reference points, resulting in:

$$
w(p)=\left(\delta\left(p-R_{i-1}\right)^{\gamma} /\left(\delta\left(p-R_{i-1}\right)^{\gamma}+\left(R_{i}-p\right)^{\gamma}\right)\right) \bullet\left(R_{i}-R_{i-1}\right) /\left(R_{n}-R_{0}\right)+R_{i-1} / R_{n}
$$

In the above, we continue to use $p$ as the expression for the probability input to differentiate it from the value input in the larger $\mathrm{CPT}$ equation. But $p$ now refers to the numerator of the ratio implied by the percentage sign (such as ' 60 ' for 60/100), rather than already being translated into a proportion. Two versions of this equation were used in the present work. In the one-cycle CPT model (a shorthand for a CPT equation with a one-cycle probability weighting function), 0 and 100 are assumed to be the only reference values used by the decision maker. The one-cycle version is functionally the same as the Lattimore et al. equation (the parts after the multiplication 
sign are identity operations in the one-cycle model) and can produce a standard probability weighting curve (an inverse S-shape) across the full 0-100 scale range (as in Fig. 3a). In a twocycle CPT model, 50 is included as an additional reference point, such that the equation can produce an inverse S-shape between 0 and 50 that is repeated between 50 and 100 (as in Fig 3b). Because there is also an elevation parameter in both versions to accommodate the asymmetrical crossover commonly seen in decision making research, any inverse S-shape need not be symmetrical to be well fit by the equation; that is, the equation accommodates crossovers in locations other than the midpoint of each S-shaped curve.

The CPT equation was thus modified as follows. We started with the general specification of the CPT model (Eq. 1) shown earlier:

$$
v(C E)=v\left(x_{1}\right) w(p)+v\left(x_{2}\right)(1-w(p)) .
$$

We then elaborated the value and probability weighting functions using the original value function (Eq. 2) and the CPM-inspired probability weighting function (Eq. 9):

$$
\begin{aligned}
& C E^{\alpha}=x_{1}^{\alpha} \cdot\left(\delta\left(p-R_{i-1}\right)^{\gamma} /\left(\delta\left(p-R_{i-1}\right)^{\gamma}+\left(R_{i}-p\right)^{\gamma}\right) \cdot\left(R_{i}-R_{i-1}\right) /\left(R_{n}-R_{0}\right)+R_{i-1} / R_{n}\right) \\
& +x_{2}^{\alpha} \cdot\left(1-\left(\delta\left(p-R_{i-1}\right)^{\gamma} /\left(\delta\left(p-R_{i-1}\right)^{\gamma}+\left(R_{i}-p\right)^{\gamma}\right) \cdot\left(R_{i}-R_{i-1}\right) /\left(R_{n}-R_{0}\right)+R_{i-1} / R_{n}\right)\right)
\end{aligned}
$$

And we raised both sides by $1 / \alpha$ to isolate the $C E$ dependent measure:

$$
\begin{gathered}
C E=\left(x_{1}^{\alpha} \cdot\left(\delta\left(p-R_{i-1}\right)^{\gamma} /\left(\delta\left(p-R_{i-1}\right)^{\gamma}+\left(R_{i}-p\right)^{\gamma}\right) \cdot\left(R_{i}-R_{i-1}\right) /\left(R_{n}-R_{0}\right)+R_{i-1} / R_{n}\right)\right. \\
\left.+x_{2}^{\alpha} \cdot\left(1-\left(\delta\left(p-R_{i-1}\right)^{\gamma} /\left(\delta\left(p-R_{i-1}\right)^{\gamma}+\left(R_{i}-p\right)^{\gamma}\right) \cdot\left(R_{i}-R_{i-1}\right) /\left(R_{n}-R_{0}\right)+R_{i-1} / R_{n}\right)\right)\right)^{1 / \alpha}
\end{gathered}
$$

As will be described in Section 1.5, we used this equation to test whether $C E$ responses made by each individual in our study were better fit by a one-cycle CPT model (Eq. 11 with $R_{0}=0$ and $R_{1}$ $=100)$ or a two-cycle CPT model $\left(R_{0}=0, R_{1}=50\right.$, and $\left.R_{2}=100\right)$ in each study condition.

\subsection{Overview of present study}

The goal of the present study was to attempt to manipulate use of two versus three 
reference points for estimating probability in a gambling task and to assess fit of one-cycle CPT versus two-cycle CPT models to individual certainty equivalent data. Specifically, participants responded to a series of 176 gambles and $C E$ data were derived from participant responses. Participants in a first sample were assigned either to a full-range condition, in which probabilities (presented as percentages) ranged from $0-100 \%$ as they typically do in tasks of this type, or to an upper-range condition, in which probabilities ranged from 50-100\%. In these conditions, participants were not explicitly told the probability range, but were expected to infer it from the gambles. In order to extend the findings to a different range and to ensure that the range was known, a new sample of participants was assigned to a lower-range condition in which probabilities ranged from $0-50 \%$, and participants were explicitly instructed that all stated probabilities would be in this range. The purpose of the range manipulation across conditions was to influence reference points. We expected individuals in the full-range condition to be most likely to use only 0 and 100 as reference points, and those in the upper-range and lower-range conditions to be more likely to use 50 as an additional reference point. In all other ways, the full-range condition and the two "limited-range" conditions were the same. For ease of presentation, the three conditions are reported as a single study. Two short numeracy scales were also administered to all participants to assess their general skills with number.

In the full-range condition, we expected the $C E$ s for most individuals to be best fit by a one-cycle CPT model (Fig. 3a). In the two limited range conditions, if intermediate reference points are not used, we expected data to also be better fit by a one-cycle CPT model. That is, $C E$ s for the lower-range condition would reflect a distortion pattern matching the lower half of the inverse S-shape of the probability weighting curve, while $C E$ 's in the upper-range condition would reflect a distortion pattern matching the upper half of the same curve. If, instead, an 
intermediate reference point is used, we expected data in the limited-range conditions to be better fit by a two-cycle CPT model (Fig. 3b). In this case, $C E \mathrm{~s}$ in the lower-range condition would reflect a single inverse S-shaped curve (the lower half of the two-cycle pattern), as would CEs in the upper-range condition (in this case, reflecting the upper half of the two-cycle pattern). We expected that such two-cycle curves, if present, would otherwise be similar to the one-cycle curve for the full-range condition. That is, the use of an additional reference point should affect the number of cycles in the probability weighting curve but not its degree of curvature or elevation (or the curvature associated with the value function; i.e., parameter estimates should not change). It is possible that there would also be some additional differences across conditions resulting from exposing participants to different ranges of probabilities (i.e., distinct from their choice of reference points), but we did not have specific predictions along these lines.

Though not the central focus, we also conducted analyses to assess the extent to which some individuals in the full-range condition might have spontaneously used a middle reference point. That is, we considered how many individuals in the full-range condition had $C E$ data better fit by a two-cycle than a one-cycle CPT model. Additionally, after conducting initial analyses, we developed a mixed-cycle CPT model to assess whether such a model might explain patterns in the data better than either a one- or a two-cycle model alone. Such a model would be expected to fit $C E$ data well if in one or more conditions a middle reference point was used on only a subset of trials. Finally, deviations of the probability weighting curve (and also the value curve) from the identity line, as well as model fitting error, were expected to be negatively correlated with numeracy. Such a finding would be consistent with past work (using a full-range condition; Patalano et al., 2015) and would provide further evidence of a relationship between probability distortion and intuitive number skill more generally. 


\section{Method}

\subsection{Participants}

A total of 137 undergraduates (56 male, 79 female, 2 not reported; 18 - 22 years old) received introductory psychology course credit or monetary compensation for their participation. Participants in a first group were randomly assigned to either the full-range $(n=44)$ or upperrange $(n=45)$ condition (with no explicit statement of probability range). A second group (run after the first group) was assigned to the lower-range $(n=48)$ condition (with explicit statement of probability range). Two additional participants came to the lab but did not complete the gambling task. Of those completing the gambling task, 9 participants were excluded from final data analysis based on the a priori elimination rule of having a large number of missing trials ( $>$ 30 out of 176 trials), and 1 additional participant was excluded for extreme root mean square error (RMSE) values (> $10 S D$ above the mean) for both CPT models. Analyses were based on the remaining 127 participants (full-range: $n=40$; upper-range: $n=42$; lower-range: $n=45 ; 1$ of the latter did not complete the numeracy scales and was excluded just from that analysis). Across conditions, there were no differences in the number of missing trials, $F(2,124)=0.87, p=$ .421 , or in response reliability for the 11 pairs of repeated gambles (see 2.2. Procedure section), $F(2,124)=0.18, p=.837$.

\subsection{Procedure}

Participants came individually to the lab for a two-hour session. Written informed consent of the participant was obtained at the start of each laboratory session. Participants then completed the gambling task for approximately 60 minutes, several tasks that were unrelated to the present one and that served as filler activities for approximately 30 minutes, and two numeracy measures for approximately 5 minutes. Participants completed the tasks individually 
at their own pace on the computer in the laboratory. An experimenter sat nearby to monitor the study, provide instructions, and answer any questions.

\subsubsection{Gambling task}

The gambling task was modeled after Gonzalez and Wu (1999; see also Tversky \& Kahneman, 1992). The task, as shown in Figures 4a and 4b (which represent a single trial), was to compare a stated gamble (e.g., " $95 \%$ chance of $\$ 100$, otherwise $\$ 0 ”)$ to each of six "surething" dollar amounts and to indicate a preference between the gamble and each sure-thing, and then to do the same with a narrower range of dollar values determined based on one's responses to the first screen. The certainty equivalent for the gamble was recorded as the midpoint between the dollar values on the second screen representing where the individual switched from preferring the sure-thing to preferring the gamble. Details of this task follow. Each participant completed 176 trials of the gambling task (165 unique trials and 11 repeated ones).

\subsubsection{Gambling stimuli}

A total of 15 pairs of dollar values were crossed with 11 probabilities to create a set of 165 unique two-outcome gambles. The pairs of dollar values were $25-0,50-0,75-0,100-0,150-$ 0, 200-0, 400-0, 800-0, 50-25, 75-50, 100-50, 150-50, 150-100, 200-100, and 200-150.

Probabilities varied by condition. In the full-range condition, the 11 probabilities (in a range between $0 \%$ and $100 \%$ ) were $1 \%, 5 \%, 10 \%, 25 \%, 39 \%, 50 \%, 61 \%, 75 \%, 90 \%, 95 \%$, and $99 \%$. In the upper-range condition, the probabilities (in a range between 50\% and 100\%) were 51\%, $53 \%, 55 \%, 63 \%, 70 \%, 75 \%, 80 \%, 87 \%, 95 \%, 97 \%$, and $99 \%$. In the lower-range condition, the probabilities (in a range between 0 and 50\%) were 1\%, 3\%, 5\%, 13\%, 20\%, 25\%, 30\%, 37\%, $45 \%, 47 \%, 49 \%$. The proportional spacing of probabilities was matched between conditions (e.g., $10 \%$ out of $100 \%$ matched $5 \%$ out of $50 \%$ ), with the exception that a few values were 
adjusted so that the number of values divisible by five would be about the same in all conditions. Eleven gambles, one at each probability level, were repeated as a reliability check, resulting in a total of 176 gambling trials. Ten pseudorandom orders of the trials were created, with no gamble appearing twice in a row.

\subsubsection{Task instructions and procedure}

Each participant read task instructions on the screen, paraphrased the instructions back to the experimenter, and had the opportunity to ask any questions before beginning the task. A printed sheet of instructions was also available for reference throughout the experiment. Each trial began with a display similar to that shown in Figure 4a. The sure-thing amounts ranged from the largest possible gamble outcome to the smallest (i.e., from $\$ 100$ to $\$ 0$ in Fig. $4 \mathrm{a}$ ), with intermediate values at equally spaced intervals. Participants were told that it was expected that they would "cross over" from preferring the sure-thing to preferring the gamble at some point for each display. When done with the first display, the participant proceeded to a follow-up display (for the same trial; they could then no longer return to the previous display), which presented a narrower range of sure-thing values (the new endpoints were the sure-thing values bracketing the crossover point from the previous display, e.g., the endpoints might be from $\$ 80$ to $\$ 60$; see Fig. $4 b)$. This procedure is one of several used to obtain $C E s$ in the decision literature. It is known to elicit more reliable responses than more direct procedures (e.g., directly asking for the overall value of the gamble; see Fox \& Poldrack, 2014, for review). The instructions were the same across conditions except that, in the lower-range condition, participants were explicitly told (and expected to repeat back to the experimenter during the paraphrasing of the instructions) that the probabilities ranged between 0 and $50 \%$. Any participants who did not spontaneously offer this range when paraphrasing the instructions was asked for it explicitly by the experimenter. A 
participant who could not accurately state the range was asked to re-read the instructions and the process was repeated (no one needed to re-read the instructions more than once).

\section{FIGURE 4 ABOUT HERE}

\subsubsection{Numeracy scales}

Lipkus, Samsa, and Rimer's (2001) 11-item numeracy scale and Weller, Dieckmann, Tusler, Mertz, Burns, and Peters' (2013) 8-item abbreviated numeracy scale were administered. The 5 items common to both scales were administered once, for a total of 14 items across both scales. All items on Lipkus et al.'s scale involve manipulating percentages, proportions, and fractions (e.g., "If the chance of getting a disease is 20 out of 100, this would be the same as having a __ $\%$ chance of getting the disease.”). The Weller et al. scale also includes several word problems that are more difficult (e.g., "If it takes five machines five minutes to make five widgets, how long would it take 100 machines to make 100 widgets?"), but that are less directly related to manipulating part-whole relationships. Paper and pencil were available during this task and participants were free to jot down any computations.

\section{Results}

\subsection{Gambling data processing}

For the gambling task, certainty equivalents were obtained for each of the 176 trials for each participant based on their choices for each of the gambles. A certainty equivalent could not be directly inferred for trials in which the participant gave the same response to every comparison (i.e. the person chose all "sure-thing" or all "gamble" responses). These trials were excluded and responses were considered missing except for ones in which a participant indicated a crossover point on the first screen of a trial. In these cases, because the certainty equivalent range was narrowed by the first response, it was reasonable to conclude that a participant who 
chose all "sure-thing" responses on the second screen was intending the lowest crossover point and a participant who chose all "gamble" responses intended the highest (see Patalano et al., 2015). As mentioned in the Participants section (Section 2.1), those participants with more than 30 missing trials were eliminated from further consideration.

For each condition, one-cycle and two-cycle modified CPT models (based on Eq. 11) were fit to $C E$ data. An $\alpha$ parameter was estimated as a measure of curvature of the value function, $\delta$ was estimated as a measure of the elevation of the probability weighting function, and $\gamma$ was estimated as a measure of curvature of the probability weighting function for each model. The modified CPT model was specified as (Eq. 11, rewritten here): $C E=\left(x_{1}^{\alpha} \bullet\left(\delta\left(p-R_{i-1}\right)^{\gamma} /(\delta(p\right.\right.$ $\left.\left.\left.-R_{i-1}\right)^{\gamma}+\left(R_{i}-p\right)^{\gamma}\right) \cdot\left(R_{i}-R_{i-1}\right) /\left(R_{n}-R_{0}\right)+R_{i-1} / R_{n}\right)+x_{2}^{\alpha} \cdot\left(1-\left(\delta\left(p-R_{i-1}\right)^{\gamma} /\left(\delta\left(p-R_{i-1}\right)^{\gamma}+\left(R_{i}-p\right)^{\gamma}\right) \bullet\right.\right.$ $\left.\left.\left.\left(R_{i}-R_{i-1}\right) /\left(R_{n}-R_{0}\right)+R_{i-1} / R_{n}\right)\right)\right)^{1 / \alpha}$. For the one-cycle version of the CPT model, $R_{0}=0$ and $R_{1}=$ 100. For the two-cycle version, $R_{0}=0, R_{1}=50$, and $R_{2}=100$. SPSS statistical software, nonlinear least squares regression, and a sequential quadratic programming algorithm for parameter estimation were used. Parameter starting values considered were $.5, .8$ and 1.1 and the starting value that resulted in the lowest RMSE (with plausible parameter values) was selected (.8 was the preferred starting value in nearly all cases). Parameter estimates were constrained to be $>0$. Resulting distributions of estimates were positively skewed (skewness $>1$ ), so medians and nonparametric statistical tests are reported unless otherwise noted. For interested readers, we also developed modified one- and two-cycle CPT models using Tversky and Kahneman's (1992) original one-parameter probability weighting function (instead of the two-parameter function) and fit these models to our data. See Supplemental Materials for details.

\subsection{Numeracy scales}


Numeracy scales were scored as the total number of correct responses. For the Lipkus et al. (2001) scale, the mean was $9.22(S D=1.33$, range $=6-11$, skewness $=-0.60)$, and for the Weller et al. (2013) scale it was $5.37(S D=1.44$, range $=2-8$, skewness $=-0.30)$. With the Lipkus et al. scale, individuals in the lower-range condition $(M=8.55)$ had lower numeracy scores than those in the full-range $(M=9.68)$ and upper-range $(M=9.50)$ conditions, $F(2,123)=$ $10.24, p<.001)$; the findings were the same with the Weller et al. scale. It is not clear why numeracy scores were lower for the lower-range condition. The participants were drawn from a different Introductory Psychology class, but one expected to have similar characteristics.

\subsection{CPT model fit and parameter estimates}

\subsubsection{Plotted $C E$ medians as a function of probability level}

To provide a general sense of $C E$ patterns (following Tversky \& Kahneman, 1992), for all gambles of the form $(x, p ; 0,1-p)$, we divided the certainty equivalent $(C E)$ by the gamble outcome $(x)$. For each probability level, the median ratio was plotted by condition as shown in Figure 5. The graphed data points can be interpreted as representing the shape of the probability weighting curve in the limited case in which the value function is the identity relationship (i.e., $\alpha$ $=1$ ). We overlaid on this graph the smooth curve that reflects the best fitting one-cycle probability weighting curve for the full-range condition (plotting gamble probability against decision weight in this case). The figure shows that $C E$ response patterns were somewhat similar across conditions, although with some deviations in the limited-range conditions. It is important to keep in mind that because the diagonal line only reflects the identity line for the probability weighting function when $\alpha=1$, the $C E$ patterns in the figure are consistent with multiple interpretations of the probability weighting function (depending on $\alpha$ ). For example, it is equally plausible that the best fitting probability weighting curve for the upper-range condition data is 
fully below the probability weighting function identity line and that $\alpha=1$ or that it is partially above the identity line and that $\alpha<1$. Because our primary interest was in estimating decision weights, all future graphs show $w(p)$ plotted against $p$ using Eq. 9 and median parameter estimates obtained through modeling. $C E \mathrm{~s}$ are not plotted again on these graphs given that $C E \mathrm{~s}$ do not provide dirct evidence of the fit of the probability weighting function.

\section{FIGURE 5 ABOUT HERE}

\subsubsection{Model fit and parameter estimates for full-range condition}

Based on much past work, we predicted that the one-cycle model would fit the full-range condition data well, and it did. The first row of Table 1 shows the median parameter estimates and the model fit based on RMSE from the full-range condition using the one-cycle model. The median parameter estimates and $R M S E$ here, nearly identical to those found in past work (Patalano et al., 2015), reflect declining value of dollars, and an over-under pattern of probability distortion with a crossover at approximately $20 \%$. In Table 1, we also report the number of participants with parameter estimates less than 1 because typical qualitative patterns for the value curvature $\alpha$, probability weighting elevation $\delta$, and probability weighting curvature $\gamma$ are reflected by estimates below 1 . Note that while the table indicates the percentage of individuals better fit by each model, data from all full-range condition participants were used to compute medians for each model (the same is true for the upper- and lower-range conditions). Also, while we include median $R^{2}$ values in the table to give a more intuitive measure of fit, individuallevel $R^{2}$ values should not be interpreted as proportion of variance explained given that it is possible for the grand mean model to fit less well than a nonlinear model to an individual's data (which would result in a negative $R^{2}$ ).

TABLE 1 ABOUT HERE 
While the one-cycle model generally provided a better fit to data than the two-cycle model in the full-range condition (based on a comparison of RMSE for each model using a Wilcoxon Signed Ranks $Z$-test, $Z=3.45, p=.001$ ), the two-cycle model did provide a better fit to the data for $25 \%$ of participants in this condition. Further, for the participants better fit by the two-cycle model, median parameter estimates $(\alpha=.98, \delta=.48, \gamma=.63, R M S E=26.42)$ were similar to medians for individuals better fit by the one-cycle model $(\alpha=.80, \delta=.55, \gamma=.43$; $R M S E=26.76$; based on a Mann-Whitney U-test, $U \mathbf{s}<1.60, p s>.100$ ), as one would expect if the only change between groups was in the use of an additional reference point (see Figure 6 for one- and two-cycle curves fit to subsets of participants; this is the only analysis that uses subsets of participants). Additionally, mean numeracy scores (using the Weller et al. 8-item scale but results with the Lipkus et al. 11-item scale were similar) were higher for participants better fit by the two-cycle model $(M=6.30, S D=0.95)$ than by the one-cycle model $(M=5.64, S D=1.47)$, though the difference was not statistically significant, $t(38)=-1.33, p=.191$. These findings, while by no means conclusive, are suggestive that some individuals, who may be more numerate, may have utilized multiple reference points.

\section{FIGURE 6 ABOUT HERE}

\subsubsection{Model fit and parameter estimates for limited-range conditions}

The primary question of interest was whether behavior in the upper-range and lowerrange conditions would be better fit by the one-cycle model (as in Figure 3a) or the two-cycle model (as in Figure 3b). To answer this question, we compared the fit of each model to individual data. The RMSE was found to be reliably lower for the two-cycle model than for the one-cycle model for both the upper-range condition $(Z=-3.07, p=.002)$ and the lower-range 
condition $(Z=-3.00, p=.003)$, providing initial evidence that the two-cycle model might provide a better fit to the data. A comparison of the RMSEs for each participant revealed that $67 \%$ of participants in the upper-range condition and $67 \%$ in the lower-range condition had data better fit by the two-cycle model. That said, differences in RMSEs were very small between models while differences in parameter estimates were large (see Table 1).

We then considered what the probability weighting curves from the limited-range conditions looked like relative to the standard one-cycle curve from the full-range condition. The best-fitting curves based on the one-cycle model, for all three conditions, are shown in Figure 7; the curves are very similar to one another. The estimated parameters for the one-cycle model for the upper- and lower-range conditions did not differ from those for the full-range condition (Kruskal-Wallis test $H \mathrm{~s}<5.50, p \mathrm{~s}>.080$; including for value curvature), consistent with what would be expected if the one-cycle model provided the best description of $C E$ behavior in all three conditions. We then plotted the best fitting curves for the two-cycle model for the two limited-range conditions and the one-cycle curve from the full-range condition (see Fig. 8a). Again, the curves from the limited-range conditions look very similar to the one-cycle curve for the full-range condition. What the figure broadly suggests is that the two-cycle curves, rather than being scaled representations of a typical one-cycle curve, might be better described as portions of the typical one-cycle curve that have been generated from a two-cycle equation (but with some deviations that might be meaningful, like some additional curve in the upper-range condition). It is thus not surprising that there were differences in the two-cycle model parameter estimates in the limited-range conditions relative to the parameter estimates for the one-cycle model in the full-range condition. That is, it is not surprising that different parameter estimates are needed to produce quite similar curves with different equations. 


\section{FIGURE 7 ABOUT HERE}

We formally compared parameter estimates from the one-cycle model in the full-range condition to the two-cycle model in each limited-range condition. In the lower-range condition, $\delta$ was higher than in the full-range condition $(U=3.58, p<.001)$, while $\alpha(U=-0.41, p=.682)$ and $\gamma(U=0.99, p=.318)$ did not differ across conditions. In the upper-range condition, parameter estimates for $\alpha(U=-3.30, p=.001)$ and $\delta(U=-3.04, p=.002)$ were lower, and $\gamma$ was higher $(U=2.92, p=.004)$, than those in the full-range condition. We also compared the lowerand upper-range conditions' two-cycle model parameter estimates directly to one another, finding that $\alpha(U=2.85, p=.004)$ and $\delta(U=5.52, p<.001)$ were larger and $\gamma$ was smaller $(U=$ $-1.95 p=.051)$ in the lower-range condition, and thus that behavior was not the same in the two limited-range conditions. Again, it appears that the two-cycle parameter estimates reproduce something close to the one-cycle curve using a two-cycle equation. That said, as indicated earlier, in both limited-range conditions, the data for most individuals were better fit by the twocycle model. Thus, there appears, at least on the surface, to have been some difference in behavior across contexts that might have resulted in the two-cycle model being the better fitting model. Alternatively, the two-cycle model might simply fit better whenever a limited range of probabilities is used during modeling (as both limited-range conditions, by design, used only half the scale of probabilities, while the full-range condition used the full scale). We tested the latter possibility in the next analysis by re-analyzing full-range condition data.

FIGURE 8 ABOUT HERE

\subsubsection{Reanalysis of full-range condition (top vs. bottom half of probabilities)}

To address whether the use of a limited range of probabilities during modeling could explain the better fit of the two-cycle models, we re-analysed the full-range condition data. We 
ran a "top-half" analysis using only the gambles from $50-100 \%$ in the full-range condition (6 different probabilities) and a "bottom-half" analysis using only the gambles from 0-50\% (6 different probabilities; we included the gambles involving 50\% in both analyses) and compared the one-cycle and two-cycle models for each subset of data (see Table 2). (We use the terms "top-half" and "bottom-half" instead of "upper-range" and "lower-range" to differentiate these analyses from those for the upper-range and lower-range conditions.) We found that, rather than the one-cycle model fitting better than the two-cycle model, there was no difference in the fit of the two models for either the top-half $(W=-1.69, p=.090)$ or the bottom-half $(W=-1.61, p=$ .107) data. Further, parameter estimates for the one-cycle and two-cycle models for each half of the data were similar to the estimates for the corresponding models applied to upper-range and lower-range condition data $(p \mathrm{~s}>.100)$. In Figure $8 \mathrm{~b}$, we plotted best fitting curves for the twocycle model for the top-half and bottom-half of the full-range condition data; these curves are very similar to the upper-range and lower-range condition curves in Figure 8a. While the fullrange condition analyses here were based on a much smaller number of gambles than other analyses (half as many), they do provide some further evidence that the pattern of behavior seen in the upper- and lower-range conditions might not have been different from that in the fullrange condition, and that the better fit of the two-cycle model might, at least in part, be related to the range of probabilities used in modeling.

\section{TABLE 2 ABOUT HERE}

\subsubsection{Further consideration of two-cycle model fit}

While there is so far little evidence of a difference in $C E$ behavior as a function of condition, the two-cycle model did fit the data better across all conditions than might be expected based on existing literature. We sought to better understand the fit of the two-cycle model in all 
conditions. Further inspection of median $C E \mathrm{~s}$ in Figure 5 suggested that the certainty equivalents generated in all conditions for gambles involving probabilities $50 \%$ might have been larger than the $C E$ values predicted by the one-cycle CPT model, and thus closer to the values predicted by a two-cycle model (which, when $\alpha=1$, are on the identity line in this figure). The presence of these high $C E$ values at the midpoint of the $0-100 \%$ probability range might at least partially explain the good fit of the two-cycle model. We formally tested whether an individual's median $C E$ was greater than the median of the $C E$ s predicted by the one-cycle model for that individual for all gambles involving the probability closest to the middle of the full probability scale $(49 \%, 50 \%$ and $51 \%$, for the lower-range, full-range, and upper-range conditions respectively). We only used gambles of the form $(x, p ; 0,1-p)$ for this analysis. We found that the one-cycle model did underpredict $C E$ values in all three conditions. In the full-range condition, the actual $C E$ of 47.63 was reliably greater than the model estimated $C E$ of $37.97(W=$ $-2.65, p=.008)$. In the upper-range condition, the actual $C E$ of 50.50 was reliably greater than the model-estimated $C E$ of $46.06(W=-2.58, p=.010)$. And, in the lower-range condition, the actual $C E$ of 49.88 was greater than the model-estimated $C E$ of $42.39(W=-4.31, p<.001)$. The $C E$ s for the three conditions were not reliably different from one another $(H=0.48, p=.785)$. It is also noteworthy that the inverse-S shaped pattern that appears in the $C E$ data in Figure 5 for gambles between 50 and 100\% in the upper-range condition is very low on the graph relative to what would be predicted if a typical one-cycle curve were simply scaled to the $50-100 \%$ region. In the two-cycle model applied to the upper-range condition, the low $C E$ values were accommodated with an unusually low (relative to other conditions and models) $\alpha$. In contrast, no large change in $\alpha$ was needed to accommodate $C E$ data in the lower half of the probability range $(0-50 \%)$ with a two-cycle model. Thus, if one were to adopt an account of the 
findings that assumed a two-cycle model, one would also have to explain why dollar values are more steeply discounted for probabilities above $50 \%$ than for probabilities below $50 \%$. That is, such a change in $\alpha$ is possible, but it is not predicted by the CPT model and thus would require some additional explanation. Such a change in $\alpha$ would, however, explain why a two-cycle model better fit the full-range condition data only when the data were split in half: only in the latter case could a different $\alpha$ be estimated for each half of the data. In the final Results subsection 3.3.5, we consider whether it might instead be possible to explain the inverse Sshaped curve in the $C E \mathrm{~s}$ in the right half of the graph (i.e., for probabilities above 50\%) as mixed use of a one- and two-cycle CPT model, rather than by assuming a change in value curvature.

\subsubsection{Reanalysis using a mixed (one-cycle and two-cycle) model}

Our final consideration was whether the data might be well fit by a mixed model (following Hollands \& Dyre, 2000) that uses one-cycle and two-cycle CPT models in some proportion specified with a weighting parameter $\omega$, where $0 \leq \omega \leq 1$, resulting in $C E=\omega$ (onecycle-CPT equation $)+(1-\omega)$ (two-cycle-CPT equation), with one set of value and probability weighting parameters used by both equations. We considered this model because it looked possible that the pattern of $C E \mathrm{~s}$ for the upper-range condition data (as shown in Figure 5) could be produced by combining a one-cycle and a two-cycle curve (without requiring a change in $\alpha$ ). The parameter estimates and measures of model fit for the mixed model are shown in Table 3 . In the full range condition, the median $\omega$ was .93 (i.e., dominant use of the one-cycle model), while $\omega$ was .01 for the upper-range condition and .27 for the lower-range condition (i.e., both indicating dominant use of the two-cycle model); there was no reliable difference in the medians for the two limited-range conditions $(U=0.81, p=.422)$. The models fit slightly better than those of best-fitting one- or two-cycle models (not surprising as the latter are subsets of the 
mixed model) but, overall, the analyses did not change our understanding of the data. Rather, the analyses confirmed that the one-cycle model best fits $C E$ data in the full-range condition, while the two-cycle model best fits data in the limited-range conditions. While it would be possible to develop more complex mixed models (e.g., with separate value curvature and probability weighting parameters for one- and two-cycle equations), developing and testing such models was beyond the scope of the present work.

\section{TABLE 3 ABOUT HERE}

\subsubsection{Numeracy correlates}

We created a deviation score for each parameter estimate in order to test whether numeracy was associated with less deviation from the identity line (following Patalano et al., 2015). We created one set of deviation measures using parameter estimates from the best-fitting model for each participant and one set using estimates from the one-cycle model only. For $\alpha$ and $\gamma$, which are exponents in the CPT model, deviation scores $\alpha_{\mathrm{d}}$ and $\gamma_{\mathrm{d}}$ were created by taking the inverse of parameter estimates greater than 1 (e.g., 2 becomes $1 / 2$ because the degree of curvature for any exponent greater than 1 is the same as that for its inverse), and then subtracting all scores from 1 (so that larger values indicate greater deviation from 1). Because $\delta$ is a factor that raises or lowers the curve rather than an exponent, the deviation score was created by simply taking the absolute value of the difference of the parameter estimate from $1\left(\delta_{\mathrm{d}}=|1-\delta|\right){ }^{3}$ Table 4 shows Spearman correlations between deviation scores and the Weller et al. (2015) and Lipkus et al. (2001) numeracy scores. Using the best-fitting model parameter estimates, we found that numeracy scale scores were generally negatively correlated with $\alpha_{\mathrm{d}}$ or $\delta_{\mathrm{d}}$. Using parameter estimates from the one-cycle model only, just value curvature deviation was negatively

\footnotetext{
${ }^{3}$ Patalano et al. (2015) used the absolute value of the difference of the parameter estimate from 1 for all three parameters; the present approach reflects a more precise mapping of curvatures above and below identity.
} 
correlated with numeracy scores (see Table 4 for details). Patalano et al. (2015) previously reported reliable numeracy correlations with $\alpha_{\mathrm{d}}$ and $\gamma_{\mathrm{d}}$ but not with $\delta_{\mathrm{d}}$, while Schley and Peters (2014; using a one-parameter probability weighting function) reported a correlation between numeracy and $\alpha$ only. The findings suggest that the relationship with $\alpha_{\mathrm{d}}$ is the more robust one, but that differences across studies might also reflect differences in modeling procedures.

We also found the correlation between numeracy scores and $R^{2}$ for both best-fitting and one-cycle models (we used $R^{2}$ rather than $R M S E$ so that values would be comparable across conditions). $R^{2}$ values were correlated with both Weller et al. numeracy scale scores $\left(r_{\mathrm{s}} \mathrm{s}=.34\right.$, $p \mathrm{~s}<.001)$, and Lipkus et al. scores $\left(r_{\mathrm{s}} \mathrm{s}=.23-.24, p \mathrm{~s}<.010\right)$. We tested whether numeracy predicted the best-fitting model (i.e., one-cycle or two-cycle) for each individual in the limitedrange conditions, but it did not $\left(\left|r_{\mathrm{s}}\right| \mathrm{s}<.13, p \mathrm{~s}>.100\right)$. Numeracy scores were moderately correlated with response reliability across repeated trials (Pearson $r_{p} \mathrm{~s}=.23-.39, p \mathrm{~s}<.010$ ); more numerate individuals gave more consistent responses. While not the primary focus of the present work, the numeracy findings provide some evidence that fluency manipulating numbers more generally (including translating between different formats for representing part-whole relationships) predicts more veridical use of numbers in decision making.

\section{Discussion}

The primary goal of the present work was to extend the proportion judgment framework and, in particular, Hollands and Dyre's cyclical power model, to the domain of decision making under risk. We attempted to influence participants' use of a middle reference point through a manipulation of probability range. We then tested whether this manipulation would result in a change in the best-fitting CPT model from one with a one-cycle probability weighting curve to one with a two-cycle probability weighting curve with similar curvature and elevation parameter 
estimates. We found that, consistent with our predictions, for the two limited-range contexts a CPT model with a two-cycle probability weighting function fit the data reliably better than one with a one-cycle function. However, counter to our predictions, the parameter estimates for the two-cycle models for the two limited-range conditions were different from one another and were different from one-cycle-model parameter estimates for the full-range condition. In other words, the estimated probability weighting curves in the upper- and lower-range conditions were not replications of a full-range curve scaled to a narrower range. Further, when we modeled top- and bottom-half of full-range condition data separately, the two-cycle model fit as well as the onecycle model, even though probability range (and thus potential use of a middle reference point) was not manipulated in this condition. Thus, overall, the evidence was not consistent with the prediction that the best fitting model in the limited-range conditions would be a two-cycle CPT model with parameter estimates similar to those of the one-cycle models.

There was one element of procedure and one element of participant characteristics that one might propose could be related to the lack of a greater difference in $C E$ s across conditions. The first is that, in the full-range and upper-range conditions, participants were not informed of the probability context at the outset. However, this was not likely consequential in that, in the full-range condition, participants would have presumably assumed a $0-100 \%$ range and, in the lower-range condition, participants were explicitly told of the $0-50 \%$ range, yet differences in behavior in these conditions were not large either. The second element was that participants in the lower-range condition were less numerate than participants in the other two conditions. However, we do not believe this was consequential either in that numeracy was not reliably correlated with which model (one- vs. two-cycle) was the better fitting model in any condition. 
Further, individuals in full-range and upper-range conditions had similar numeracy scores, yet there was also little difference in behavior between these two conditions.

The present findings do not rule out the possibility that distortion arises from proportion judgment or, specifically, that it arises from the comparison of targets to reference points as predicted by the CPM. However, they do, at a minimum, suggest that it might not be possible (or at least might not be easy) to manipulate probability-scale reference points. Unlike the bounding reference points in a number line task (which are typically arbitrary, determined by the experimenter, and visually present on each trial), probability range is more fixed in people's minds (and cued by the percentage symbol itself); the end points have important qualitative meaning (i.e., certainty); and the range is in one's mind rather than embodied in a physical scale. Further, it is possible that the manipulation was unsuccessful here because even in the limitedrange conditions participants ultimately needed to think about probabilities from 0 to $100 \%$ (i.e., across the full range) in order to evaluate the subset of gambles that had two non-zero outcomes. That is, if one gamble offered a $10 \%$ chance of $\$ 100$, otherwise $\$ 50$, a decision maker might explicitly bring $90 \%$ (the probability associated with the second outcome) to mind in thinking about the gamble. While not all gambles included non-zero second outcomes, the presence of even a small number of them might have had an influence on the establishment of reference points in this context.

Broadly speaking, the shape of the probability weighting function has been shown to be influenced by context in other research. Most relevant to the present work, Müller-Trede, Sher, and McKenzie (2018) recently demonstrated that probability distortion patterns depend on the scale used to communicate uncertainty. Using a bounded scale for outcome values and an unbounded scale for different levels of uncertainty (the opposite of what is standard), they found 
that distortion patterns reversed; there was an inverse S-shape for the value function and, for probabilities, there was evidence of a diminishing sensitivity to probabilities. Müller-Trede et al.'s (2018) work illustrates that probability distortion is at least in part determined by whether or not the likelihood scale is bounded, a finding consistent with a proportion judgment approach, and suggests that use of intermediate reference points might be more likely to emerge in the context of novel likelihood scales.

Stewart et al.'s (2006; Stewart et al., 2015; but see Alempaki, in press) decision by sampling theory is also relevant to the present work in that, by this account, the probability weighting function is not the result of an internal mapping of probabilities to decision weights but rather is the result of a series of ordinal comparisons to probabilities sampled from the decision environment, with one's interpretation of a probability highly influenced by what other probabilities have been recently experienced. The modified CPT model developed here is similar to decision by sampling in that both approaches give some role to the decision environment on one's interpretation of a probability. However, in the decision by sampling approach, the decision weight is fully constructed through a series of binary, ordinal comparisons between the present probability and others in the decision environment. The present approach differs in that even though the context here can influence choice of reference points, the underlying process is still assumed to be a psychophysical transformation in which the shape of the curve arises from a part-whole comparison involving imprecisely-mapped magnitudes (although the present approach, of course, does not preclude the possibility that there are other contextual influences as well).

Even though the probability range manipulation did not alter the probability weighting curve in the expected manner, it was surprising that the two-cycle model fit better than (or at 
least as well as) the one-cycle curve here in a number of situations. We do not necessarily take this as evidence of the use of multiple reference points or of generally better fit of two-cycle models. However, we do take it as evidence that there might have been some aspects of $C E$ behavior that were not fully consistent with a one-cycle model, and that were better fit by the two-cycle model in some circumstances. In particular, in the $C E$ data, there was evidence of overestimation (relative to the one-cycle model prediction) of probabilities at $50 \%$, and there was evidence of an additional inverse S-shaped curve in the upper-range of the scale. To assess whether the full-range condition findings extended to other data sets, we conducted a new analysis in which we fit one-cycle and two-cycle models to a different set of full-range data from our lab that used more common probability values $(1 \%, 5 \%, 10 \%, 25 \%, 40 \%, 50 \%, 60 \%, 75 \%$, $90 \%, 95 \%, 99 \%)$. The pattern of findings was the same, with the one-cycle model providing better overall fit and the two-cycle model providing a better fit to each half of these full-range data. What we suspect is that the two-cycle model fits the data slightly better in contexts in which the two described properties (i.e., overestimation at around 50\% and inverse S-shaped curvature in the upper range) have greater influence during modeling, such as when a greater proportion of the data points are close to 50\% (see Alempaki et al., in press, for probabilityrange-related issues in modeling).

Even though we ultimately conclude that there there is little evidence in support of the two-cycle model, it does appear that probabilities at 50\% were overweighted by participants relative to the predictions of a one-cycle CPT model. One possible reason is that individuals have a better understanding of $50 \%$ (and perhaps numbers that round to it, such as $55 \%$ ) due to familiarity (e.g., coin flips), or that the equal likelihood of two events might hold a special status in the mind - perhaps a weaker version of the type of status afforded to complete certainty (i.e., 
$0 \%$ and $100 \%$ ). Even if $50 \%$ is not used as a reference point in the manner described by proportion judgment models such as the CPM, it might nonetheless be used as an additional anchor (like 0 and $100 \%$ ) to which other probabilities are compared. It is also possible that there are unexplored demand characteristics of the present elicitation procedure that contributed to the pattern of data, although, as indicated earlier, the elicitation method is a frequently used one. We have not encountered a similar pattern in past work (e.g., Gonzalez \& Wu, 1999; Tversky \& Kahenman, 1992) but it is also not common that median CEs are reported as a function of probability level. The data pattern seems worth some further investigation.

An unresolved question is whether there might be a probability weighting function that fits the data better than either the one-cycle or the two-cycle model but that does not require different value parameters (in the case of the two-cycle model) for the upper and lower halves of the probability scale. In the present work, we considered the mixed (one- and two-cycle) model, but this did not offer new insight. One possibility for consideration for future work would be a model similar to the present two-cycle model in which the curvature of the probability weighting function is reference-point dependent, but where the elevation of the curve is not. To be concrete, imagine plotting a symmetrical two-cycle inverse S-shaped curve, and then raising or lowering the entire curve (constrained only by the 0 and $100 \%$ endpoints). It is not clear if such a model would fit the data better, but the approach would arguably be more consistent with the interpetation of the elevation parameter as being related to the overall attractiveness of gambling rather than to proportion judgment. With such an approach, it would be possible to produce a curve that falls largely below (or above) the identity line yet is still multi-cyclical, and it would be possible for the decision weight associated with $50 \%$ to take a value other than .50 (in the present two-cycle model, it will always be .50). 
The present work offers the first study aimed at testing a modified cumulative prospect theory model with a cyclical probability weighting function. Although the data do not provide compelling support for the modified CPT model, they do illustrate that distortion is not highly influenced by simple manipulations of probability range. For example, the underweighting of a probability such as $70 \%$ appears to occur whether the probability appears in the context of probabilities between $0-100 \%$ or between $50-100 \%$. Another avenue for future work is the consideration of stronger efforts to manipulate reference points including using decision tasks in which narrower boundaries more naturally emerge from the given context (e.g., if you knew that mutually-exclusive Outcome A were more likely than Outcome B, it would be more natural to then assume that Outcome A must have at least a 50\% chance); using more extensive training regarding probability context (e.g., learning the likelihood of a new rare disease over many sessions); following Müller-Trede et al. (2018), using unfamiliar likelihood scales (e.g., a scale from 0-10 where 0 means definitely will not happen, and 10 means equally likely to happen or not happen); using probability wheels (circular representations with pie-shaped portions) to represent probabilities visually; and even re-doing the present task using only $\$ 0$ as the outcome for the second gamble. It is important to continue to pursue whether there are unifying cognitive explanations for similar patterns of probability distortion across tasks. Although the present study highlights some ways in which decision making under risk might be different from other proportion judgment tasks, proportion judgment remains a promising candidate as a cognitive account of probability distortion in decision making. 


\section{References}

Alempaki, D., Canic, E., Mullett, T. L., Skylark, W. J., Starmer, C., Stewart, N., \& Tufano, F. (in press). Reexamining how utility and weighting functions get their shapes: A quasiadversarial collaboration providing a new interpretation. Management Science.

Barth, H., \& Paladino, A. M. (2011). The development of numerical estimation: Evidence against a representational shift. Developmental Science, 14, 125-135.

Barth, H., Lesser, E., Taggart, J., \& Slusser, E. B. (2015). Spatial estimation: a non-Bayesian alternative. Developmental Science, 18, 853-862.

Cavagnaro, D. R., Pitt, M. A., Gonzalez, R., \& Myung, J. I. (2013). Discriminating among probability weighting functions using adaptive design optimization. Journal of Risk and Uncertainty, 47, 255-289.

Cohen, D. J., \& Blanc-Goldhammer, D. (2011). Numerical bias in bounded and unbounded number line tasks. Psychonomic Bulletin \& Review, 331-338.

Cohen, D. J., \& Sarnecka, B. (2014). Children's number-line estimation shows development of measurement skills (not number representations). Developmental Psychology, 50, 16401652.

Fox, C. R., \& Poldrack, R. A. (2014). Prospect theory and the brain. In P. Glimcher \& E. Fehr (Eds.), Handbook of Neuroeconomics (2nd ed., pp. 533-567). New York: Elsevier.

Glöckner, A., \& Pachur, T. (2012). Cognitive models of risky choice: Parameter stability and predictive accuracy of prospect theory. Cognition, 123, 21-32.

Goldstein, W. M., \& Einhorn, H. J. (1987). Expression theory and the preference reversal phenomena. Psychological Review, 94, 236-254. 
Gonzalez, R., \& Wu, G. (1999). On the shape of the probability weighting function. Cognitive Psychology, 38, 129-166.

Hogarth, R. M., \& Einhorn, H. J. (1990). Venture theory: A model of decision weights. Management Science, 36, 780-803.

Hollands, J. G., \& Dyre, B. (2000). Bias in proportion judgments: The cyclical power model. Psychological Review, 107, 500-524.

Kahneman, D., \& Tversky, A. (1984). Choices, values, and frames. American Psychologist, 39, 341-350.

Klein, R. A., Vianello, M., Hasselman, F., Adams, B. G., Adams, R. B., Jr., Alper, S....Nosek, B. A. (2018). Many labs 2: Investigating variation in replicability across samples and settings. Advances in Methods and Practices in Psychological Science, 1, 443-490.

Lattimore, P. K., Baker, J. R., \& Witte, A. D. (1992). The influence of probability on risky choice: A parametric examination. Journal of Economic Behavior and Organization, 17, $377-400$.

Libertus, M. E., \& Brannon, E. M. (2009). Behavioral and neural basis of number sense in infancy. Current Directions in Psychological Science, 18, 346-351.

Link, Huber, S., Nuerk, H.-C., \& Moeller, K. (2014). Unbounding the mental number line: New evidence on children's spatial representation of numbers. Frontiers in Psychology, 4, 1021.

Lipkus, I. M., Samsa, G., \& Rimer, B. K. (2001). General performance on a numeracy scale among highly educated samples. Medical Decision Making, 21, 37-44. 
Müller-Trede, J., Sher, S., \& McKenzie, C. R. M. (2018). When payoffs look like probabilities: Separating form and content in risky choice. Journal of Experimental Psychology: General, 147, 662-670.

Patalano, A. L., Saltiel, J. R., Machlin, L., \& Barth, H. (2015). The role of numeracy and approximate number system acuity in predicting value and probability distortion. Psychological Bulletin and Review, 22, 1820-1829.

Peters, E., Slovic, P., Västfjäll, D., \& Mertz, C. K. (2008). Intuitive numbers guide decisions. Judgment and Decision Making, 3, 619-635.

Reyna, V. F., Nelson, W. L., Han, P. K., \& Dieckmann, N. F. (2009). How numeracy influences risk comprehension and medical decision making. Psychological Bulletin, 135, 943-973.

Rottenstreich, Y., \& Hsee, C. S. (2001). Money, kisses, and electric shocks: On the affective psychology of risk. Psychological Science, 12, 185-190.

Rouder, J. N., \& Geary, D. C. (2014). Children's cognitive representation of the mathematical number line. Developmental Science, 17, 525-536.

Schley, D. R., \& Peters, E. (2014). Assessing economic value: Symbolic-number mappings predict risky and riskless valuations. Psychological Science, 25, 753-761.

Slusser, E., \& Barth, H. (2017). Intuitive proportion judgment in number-line estimation: Converging evidence from multiple tasks. Journal of Experimental Child Psychology, 70, 181-198.

Slusser, E., Santiago, R., \& Barth, H. (2013). Developmental change in numerical estimation. Journal of Experimental Psychology: General, 142, 193-208.

Spence, I. (1990). Visual psychophysics of simple graphical elements. Journal of Experimental Psychology: Human Perception and Performance, 16, 683-692. 
Stevens, S. S. (1957). On the psychophysical law. Psychological Review, 64, 153-181.

Stewart, N., Chater, N., \& Brown, G. D. (2006). Decision by sampling. Cognitive Psychology, $53,1-26$.

Stewart, N. (2009). Decision by sampling: The role of the decision environment in risky choice. Quarterly Journal of Experimental Psychology, 62, 1041-1062.

Stewart, N., Reimers, S., Harris, A. J. L. (2015). On the origin of utility, weighting, and discounting functions: How they get their shapes and how to change their shapes. Management Science, 61, 687-705.

Stott, H. P. (2006). Cumulative prospect theory's functional menagerie. Journal of Risk and Uncertainty, 32, 101-130.

Sullivan, J., Juhasz, B., Slattery, T., \& Barth, H. (2011). Adults' number-line estimation strategies: Evidence from eye movements. Psychonomic Bulletin \& Review, 18, 557-563.

Tversky, A., \& Kahneman, D. (1992). Advances in prospect theory: Cumulative representation of uncertainty. Journal of Risk and Uncertainty, 5, 297-323.

Ungemach, C., Stewart, N., \& Reimers, S. (2011). How incidental values from our environment affect decisions about money, risk, and delay. Psychological Science, 22, 253-260.

Varey, C. A., Mellers, B. A., \& Birnbaum, M. H. (1990). Judgments of proportions. Journal of Experimental Psychology: Human Perception and Performance, 16, 613-625.

Wedell, D. H. (1991). Distinguishing among models of contextually induced preference reversals. Journal of Experimental Psychology: Learning, Memory, and Cognition, 17, $767-778$. 
Weller, J. A., Dieckmann, N. F., Tusler, M., Mertz, C. K., Burns, W. J., \& Peters, E. (2013). Development and testing of an abbreviated numeracy scale: A Rasch analysis approach. Journal of Behavioral Decision Making, 26, 198-212.

Windschitl, P. D., \& Chambers, J. R. (2004). The dud-alternative effect in likelihood judgment. Journal of Experimental Psychology: Learning, Memory, and Cognition, 30, 198-215.

Winman, A., Juslin, P., Lindzog, M., Nilsson, H., \& Kerimi, N. (2014). The role of ANS acuity and numeracy for the calibration and the coherence of subjective probability judgments. Frontiers in Psychology, 5. doi: 10.3389/fpsyg.2014.00851

Zax, A., Slusser, E. B., \& Barth, H. (2018). Partitioning and proportion estimation in children's numerical judgments. Manuscript in preparation.

Zhang, H., \& Maloney, L. T. (2012). Ubiquitous log odds: A common representation of probability and frequency distortion in perception, action, and cognition. Frontiers in Neuroscience, 6:1. doi:10.3389/fnins.2012.00001 
Table 1. Median parameter estimates and model fit measures for one-cycle versus two-cycle CPT model fit to individual-level data in each condition

\begin{tabular}{lcccccc}
\hline Condition & Model & $\begin{array}{r}\text { Ps best fit } \\
\text { by model }\end{array}$ & $\begin{array}{c}\text { Value } \\
\text { curvature } \\
(\alpha)\end{array}$ & $\begin{array}{c}\text { Probability } \\
\text { weighting } \\
\text { elevation } \\
(\delta)\end{array}$ & $\begin{array}{c}\text { Probability } \\
\text { weighting } \\
\text { curvature } \\
(\gamma)\end{array}$ & $R M S E$ \\
\hline $\begin{array}{l}\text { Full- } \\
\text { range } \\
(n=40)\end{array}$ & one-cycle & $75 \%$ & $.80(76 \%)$ & $.61(81 \%)$ & $.57(89 \%)$ & $26.76(.91)$ \\
\hline $\begin{array}{l}\text { Upper- } \\
\text { range } \\
(n=42)\end{array}$ & one-cycle & $33 \%$ & $.90(72 \%)$ & $.69(84 \%)$ & $.46(91 \%)$ & $28.15(.91)$ \\
\hline $\begin{array}{l}\text { Lower- } \\
\text { range } \\
(n=45)\end{array}$ & one-cycle & $33 \%$ & $.74(91 \%)$ & $.82(69 \%)$ & $.51(87 \%)$ & $20.54(.89)$ \\
\hline
\end{tabular}

Notes: Data from all participants within a condition were used to produce descriptive statistics (not just one's best fit by each model). In parentheses with each parameter estimate is the percentage of participants with estimate $<1$. In parentheses with $R M S E$ is $R^{2}$ value. Two-cycle model fit individual data better than one-cycle model in both upper-range and lower-range conditions $(p \mathrm{~s}<.05)$. However, the models did not differ substantively in their $C E$ predictions (see Fig. 7). 
Table 2. Median parameter estimates and model fit measures for one-cycle versus two-cycle CPT model fit to individual-level data for top (50-100\%) versus bottom (0-50\%) halves of probabilities of full-range condition

\begin{tabular}{lcccccc}
\hline $\begin{array}{l}\text { Full- } \\
\text { range } \\
\text { condition } \\
\text { subset }\end{array}$ & Model & $\begin{array}{c}\text { Ps best } \\
\text { fit by } \\
\text { model }\end{array}$ & $\begin{array}{c}\text { Value } \\
\text { curvature } \\
(\alpha)\end{array}$ & $\begin{array}{c}\text { Probability } \\
\text { weighting } \\
\text { elevation } \\
(\delta)\end{array}$ & $\begin{array}{c}\text { Probability } \\
\text { weighting } \\
\text { curvature } \\
(\gamma)\end{array}$ & RMSE \\
\hline $50-100 \%$ & one-cycle & $40 \%$ & $.91(58 \%)$ & $.57(88 \%)$ & $.58(88 \%)$ & $28.18(.92)$ \\
$50-100 \%$ & two-cycle & $60 \%$ & $.66(75 \%)$ & $.27(90 \%)$ & $.88(65 \%)$ & $28.55(.92)$ \\
\hline $0-50 \%$ & one-cycle & $35 \%$ & $.74(78 \%)$ & $.67(83 \%)$ & $.55(80 \%)$ & $19.11(.90)$ \\
$0-50 \%$ & two-cycle & $65 \%$ & $.71(85 \%)$ & $1.21(30 \%)$ & $.61(85 \%)$ & $16.70(.90)$ \\
\hline
\end{tabular}

$n=40$. Notes: One-cycle model did not fit individual data better than two-cycle model using either top- or bottom-half of data $(p s>.08)$. Parameter estimates here were similar to one- and two-cycle model estimates for upper-range and lower-range conditions (reported in Table 1). 
Table 3. Median parameter estimates and model fit measures for mixed (weighted one-cycle and two-cycle) CPT model fit to individual-level data in each condition

\begin{tabular}{lcccccc}
\hline Condition & Model & $\begin{array}{c}\text { Weight } \\
(\omega)\end{array}$ & $\begin{array}{c}\text { Value } \\
\text { curvature } \\
(\alpha)\end{array}$ & $\begin{array}{c}\text { Probability } \\
\text { weighting } \\
\text { elevation } \\
(\delta)\end{array}$ & $\begin{array}{c}\text { Probability } \\
\text { weighting } \\
\text { curvature } \\
(\gamma)\end{array}$ & $R M S E$ \\
\hline Full-range & mixed & 0.93 & $.87(70 \%)$ & $.47(85 \%)$ & $.59(83 \%)$ & $26.33(.91)$ \\
Upper-range & mixed & 0.01 & $.68(79 \%)$ & $.32(88 \%)$ & $.79(71 \%)$ & $27.42(.91)$ \\
Lower-range & mixed & 0.27 & $.84(84 \%)$ & $.84(56 \%)$ & $.54(82 \%)$ & $19.98(.89)$ \\
\hline
\end{tabular}

Notes: Weighting parameter $\omega$ ranges from 0 (full use of two-cycle model) to 1 (full use of onecycle model). $\omega$ did not differ reliably between upper- and lower-range conditions $(p>.100)$. The mixed models support dominant use of a one-cycle model in the full-range condition (using all data) and dominant use of a two-cycle model in the limited-range conditions. 
Table 4. Spearman correlations between deviation scores and numeracy measures

\begin{tabular}{lcccc}
\hline & $\begin{array}{c}\text { Value } \\
\text { curvature } \\
\text { deviation } \\
\left(\alpha_{\mathrm{d}}\right)\end{array}$ & $\begin{array}{c}\text { Probability } \\
\text { weighting } \\
\text { elevation } \\
\text { deviation } \\
\left(\delta_{\mathrm{d}}\right)\end{array}$ & $\begin{array}{c}\text { Probability } \\
\text { weighting } \\
\text { curvature } \\
\text { deviation } \\
\left(\gamma_{\mathrm{d}}\right)\end{array}$ & $R^{2}$ \\
\hline Weller et al. numeracy & $-.13 /-.19 *$ & $-.20 * /-.08$ & $-.05 /-.13$ & $.34 * * * / .34 * * *$ \\
Lipkus et al. numeracy & $-.20 * /-.20 *$ & $-.02 /-.01$ & $-.08 /-.16$ & $.24 * * / .23 * *$ \\
\hline
\end{tabular}

$N=126 .{ }^{* * *} p<.001 ;{ }^{* *} p<.01 ;{ }^{*} p<.05$. Notes: First value in cell is correlation between numeracy measure and deviation score for best fitting model (one-cycle or two-cycle); second score assumes one-cycle model. Additionally, $r=.65$ between numeracy scales. 


\section{Figure Captions}

Figure 1. (a) Value function $v(x)=x^{\alpha}$ with $\alpha=.77$ (black curve; for gains only). (b) Probability weighting function $w(p)=\delta p^{\gamma} /\left(\delta p^{\gamma}+(1-p)^{\gamma}\right)$ with $\delta$ (elevation) $=.77$, and $\gamma($ curvature $)=.53$

(black curve; medians from Patalano et al., 2015). (c) Probability weighting function where $\delta$ is held constant (at $\delta=1$ ) and only $\gamma$ varies across curves (resulting in a change in curvature). (d) Probability weighting function where $\gamma$ is held constant (at $\gamma=.53$ ) and only $\delta$ varies across curves (resulting in a change in elevation).

Figure 2. Curves generated using Hollands and Dyre's (2000) cyclical power model (for all curves, $\gamma=.40$ ), including a one-cycle curve, a two-cycle curve (which assumes use of an additional middle reference point); and a mixed one-cycle and two-cycle pattern (with equal weight given to each pattern). Unlike probability weighting curves in Figure 1, one- and twocycle curves here are always symmetrical around .5.

Figure 3. (a) One-cycle probability weighting curve which assumes that reference points at the boundaries of the scale are used for proportion judgment. (b) Two-cycle probability weighting curve which assumes the use of an additional middle reference point.

Figure 4. In the gambling task, participants were instructed to choose a preference between a gamble and each sure-thing option presented. Each trial consisted of: (a) an initial display (with example responses shown as Xs here) and (b) a follow-up display with sure-thing values determined by responses to the initial display. In this example, the $C E$ for this gamble would be 
70 , because this is the midpoint of the dollars where the participant "crossed over" from preferring a sure-thing to preferring a gamble on the second display.

Figure 5. Following Tversky and Kahneman (1992), for all gambles of the form $(x, p ; 0,1-p)$, the ratio of the certainty equivalent $(C E)$ to the outcome $(x)$ was calculated. For each probability level, the median ratio was plotted on the graph (by condition). The graphed data points (by condition) can be interpreted as representing the shape of the probability weighting function in the case in which the value function is the identity relationship. The smooth curve is the estimated one-cycle-model probability weighting curve for the full-range condition.

Figure 6. Best-fitting probability weighting curves for the full-range condition for individuals better fit by the one-cycle CPT model $(n=30)$ versus the two-cycle CPT model $(n=10)$. The figure illustrates that the curves can be well-described as a single inverse S-shaped curve and a two-cycle version of a curve that looks very similar to the first one. All probability weighting curves are plotted using Eq. 9, with $\delta$ and $\gamma$ replaced with median estimates.

Figure 7. Best-fitting probability weighting curves for the one-cycle CPT model for the lowerrange, upper-range, and full-range conditions.

Figure 8. Both graphs include a standard one-cycle full-range condition curve (from Fig. 7) for comparison. (a) Best-fitting probability weighting curves for the two-cycle CPT model for the lower-range and upper-range conditions. (b) Best-fitting probability weighting curves for the two-cycle CPT model for the bottom-half and top-half of the full-range condition data. Taken 
Probability range and distortion 49

together, the graphs illustrate that the two-cycle limited-range condition curves and the half-data full-range condition curves are very similar to one another. 


\section{Figure 1}

(a)

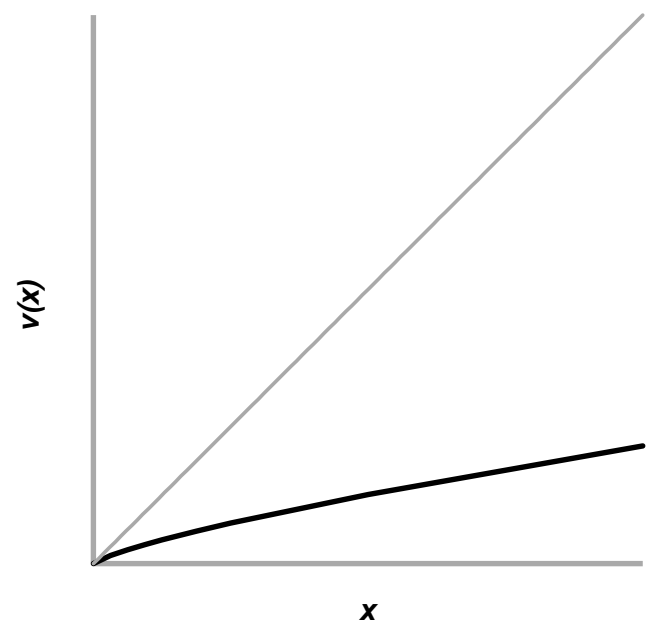

(c)

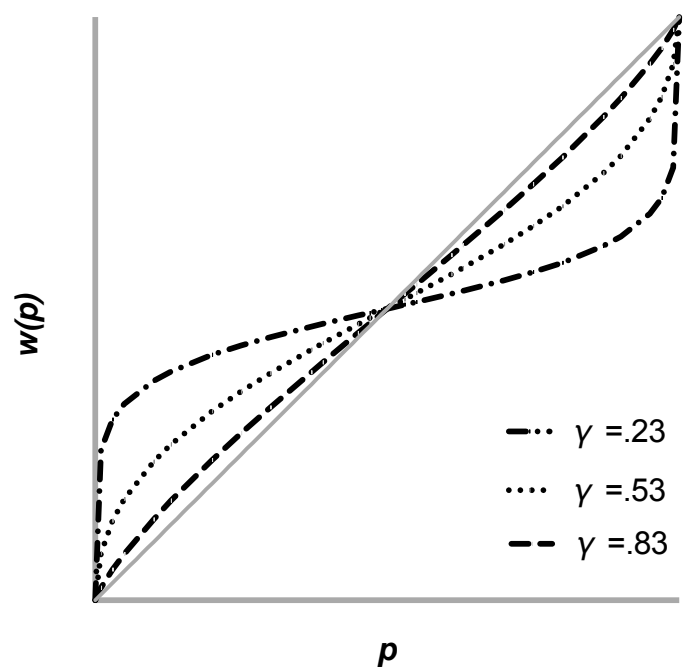

(b)

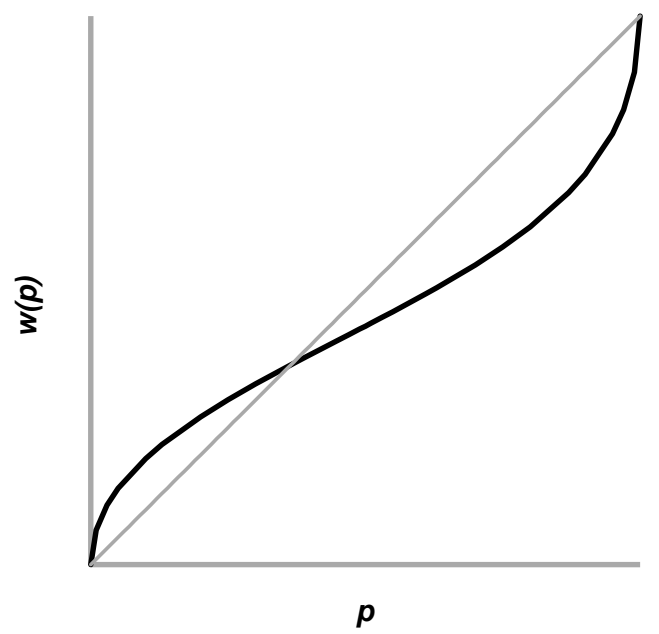

(d)

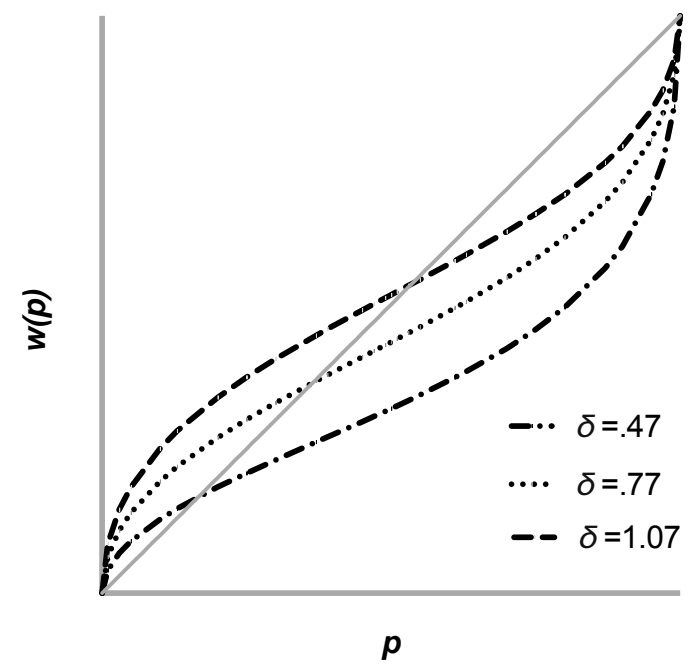


Probability range and distortion 51

\section{Figure 2}

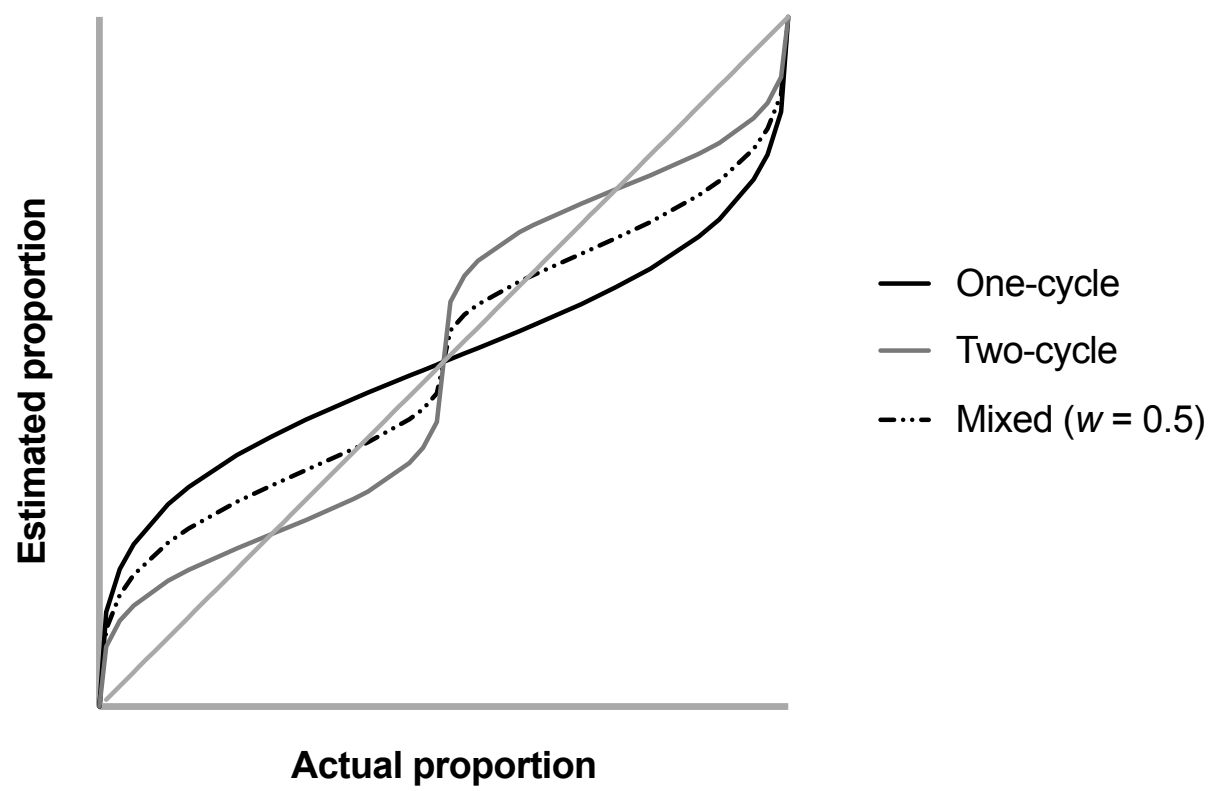


Probability range and distortion 52

\section{Figure 3}

(a)

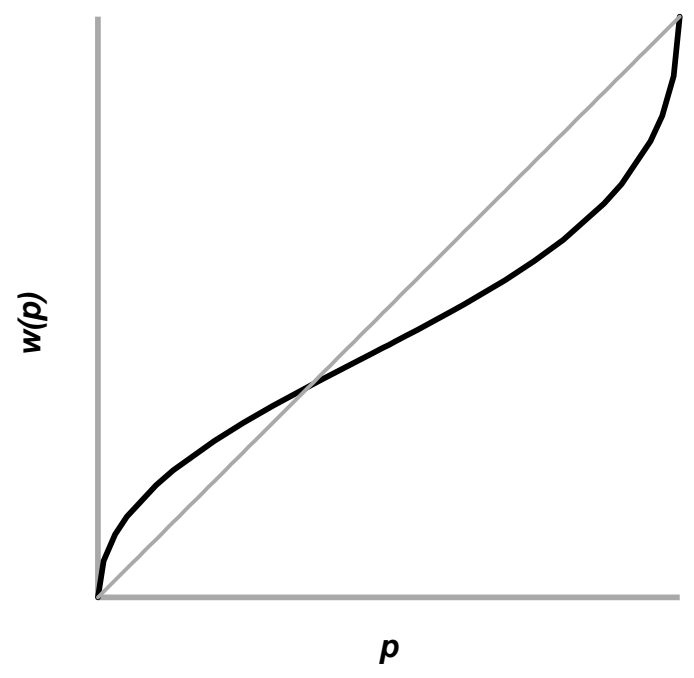

(b)

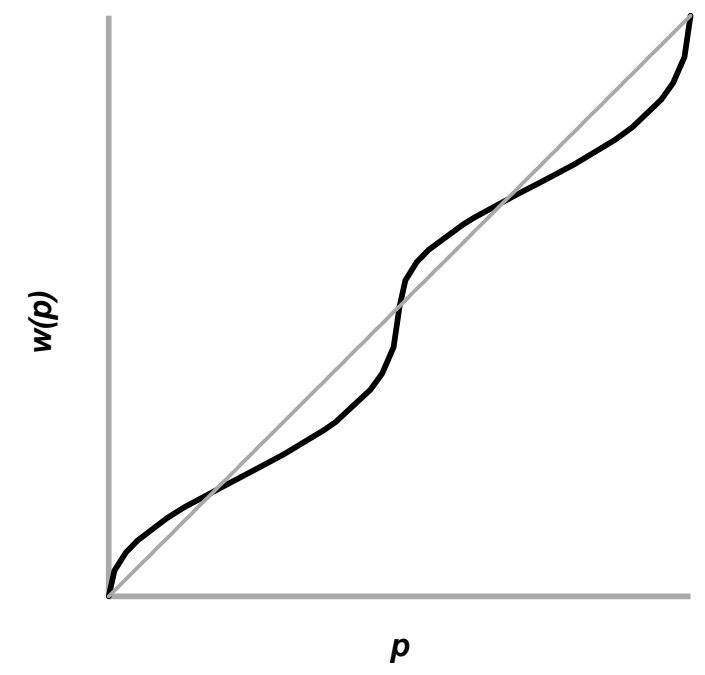


Probability range and distortion 53

\section{Figure 4}

(a)

Gamble: $95 \%$ chance of $\$ 100$, otherwise $\$ 0$

\begin{tabular}{|c|c|c|}
\hline Sure Thing & $\begin{array}{c}\text { Prefer } \\
\text { Sure Thing }\end{array}$ & $\begin{array}{c}\text { Prefer } \\
\text { Gamble }\end{array}$ \\
\hline$\$ 100$ & $\mathrm{x}$ & \\
\hline$\$ 80$ & $\mathrm{X}$ & \\
\hline$\$ 60$ & & $\mathrm{X}$ \\
\hline$\$ 40$ & & $\mathrm{x}$ \\
\hline$\$ 20$ & & $\mathrm{x}$ \\
\hline$\$ 0$ & & $\mathrm{x}$ \\
\hline
\end{tabular}

NEXT (b)

\begin{tabular}{|c|c|c|}
\hline \multicolumn{3}{|c|}{ Gamble: $95 \%$ chance of $\$ 100$, otherwise $\$ 0$} \\
\hline Sure Thing & $\begin{array}{c}\text { Prefer } \\
\text { Sure Thing }\end{array}$ & $\begin{array}{c}\text { Prefer } \\
\text { Gamble }\end{array}$ \\
\hline$\$ 80$ & $\mathrm{x}$ & \\
\hline$\$ 76$ & $\mathrm{x}$ & \\
\hline$\$ 72$ & $\mathrm{X}$ & \\
\hline$\$ 68$ & & $\mathrm{x}$ \\
\hline$\$ 64$ & & $\mathrm{x}$ \\
\hline$\$ 60$ & & $\mathrm{x}$ \\
\hline \multicolumn{3}{|c|}{ DONE } \\
\hline
\end{tabular}


Figure 5

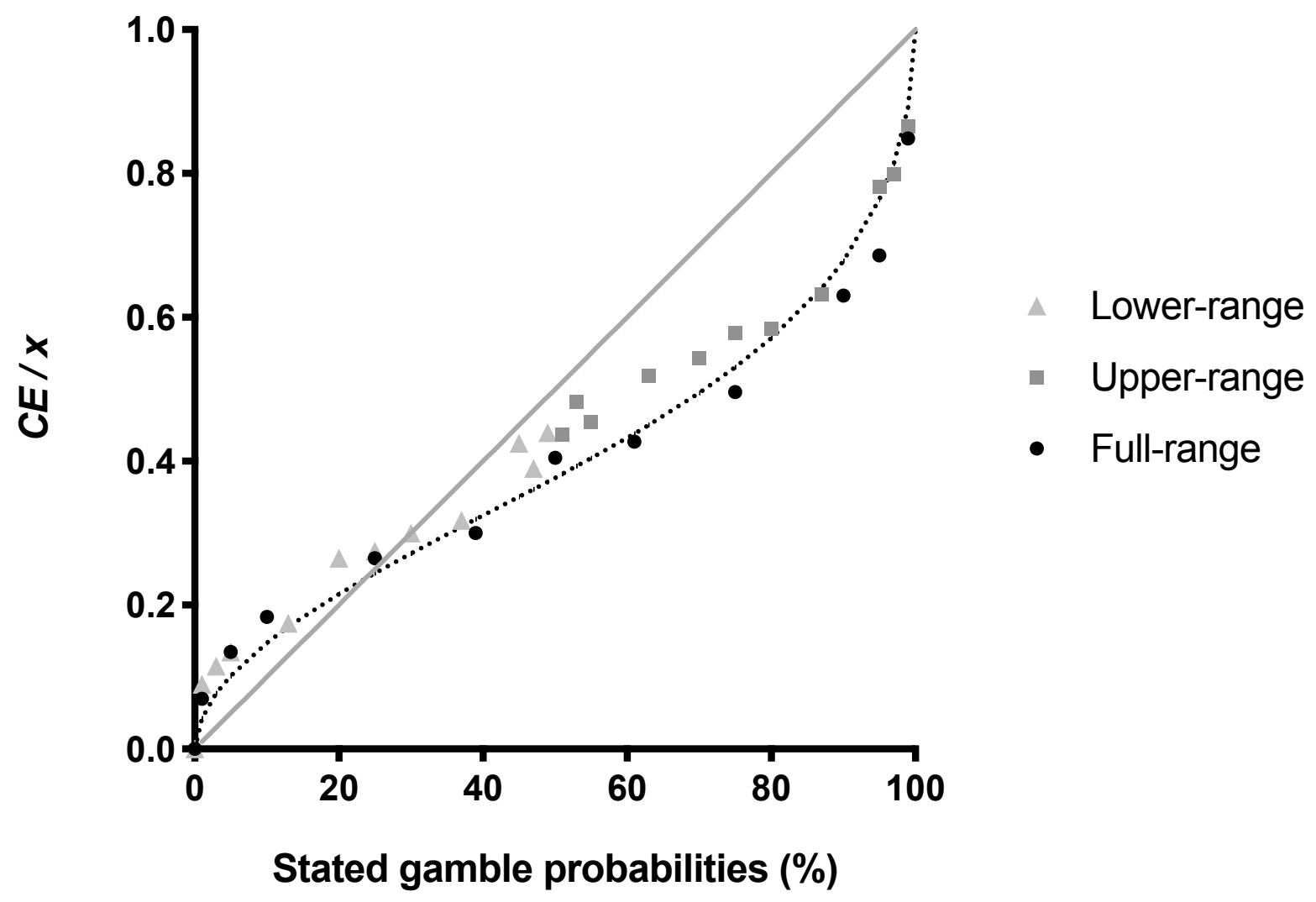


Probability range and distortion 55

\section{Figure 6}

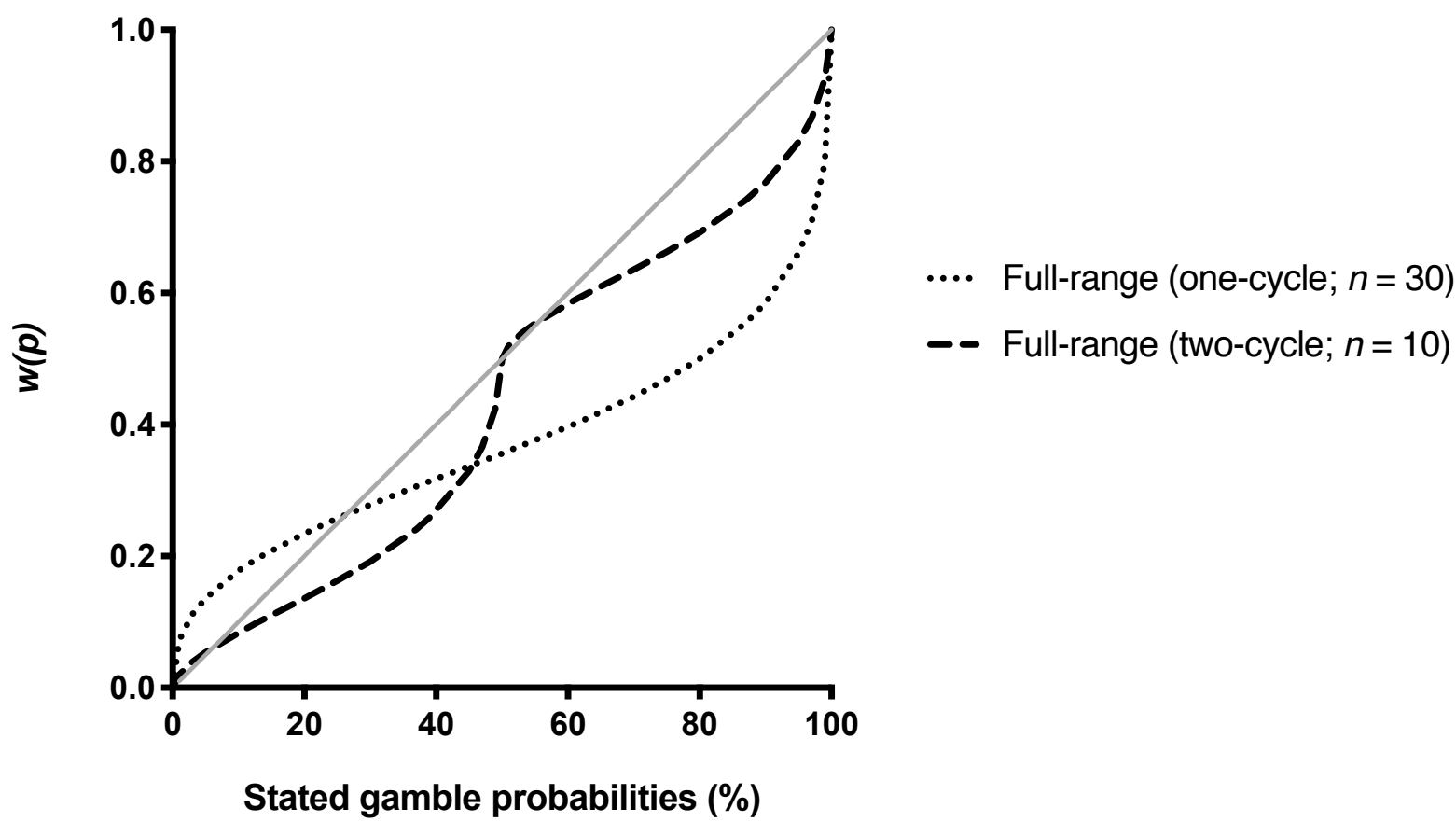




\section{Figure 7}

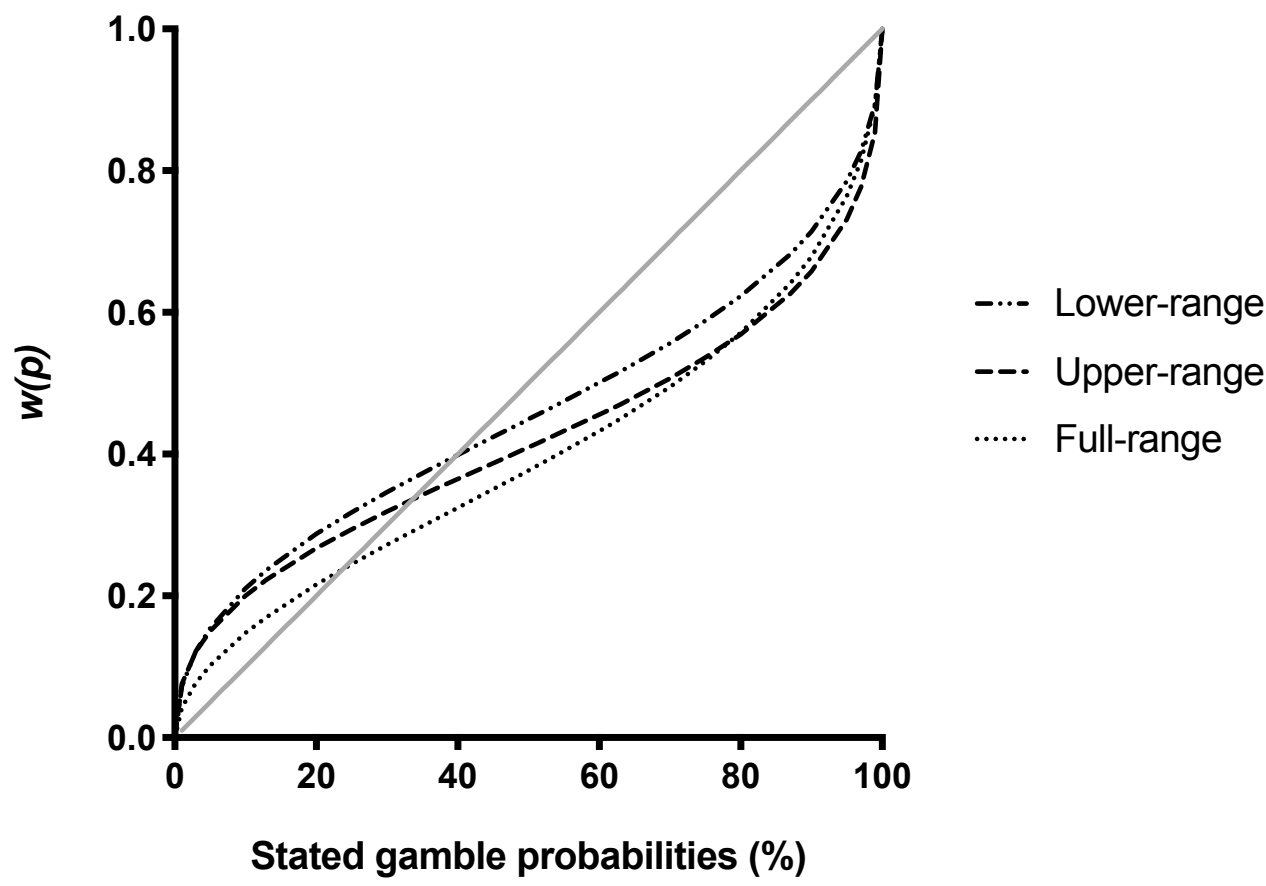




\section{Figure 8}

(a)

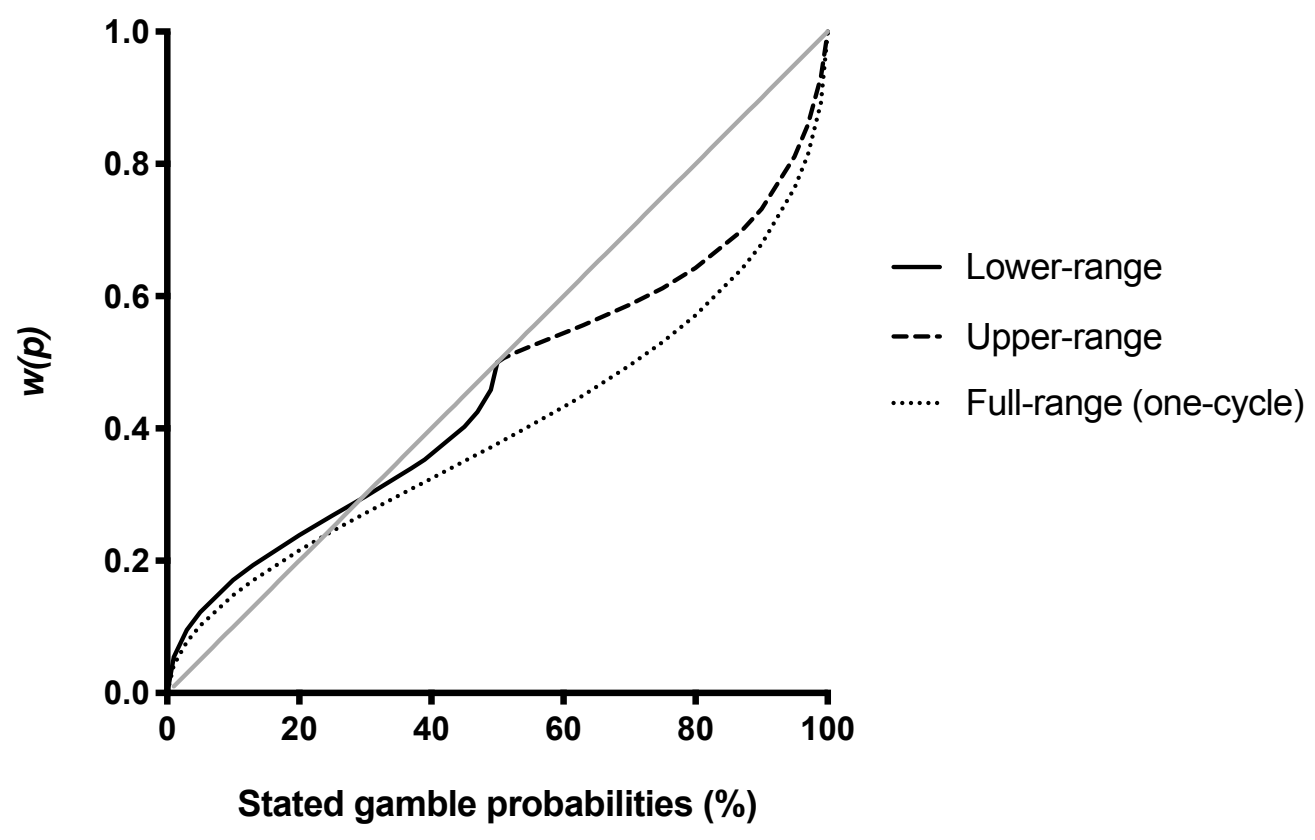

(b)

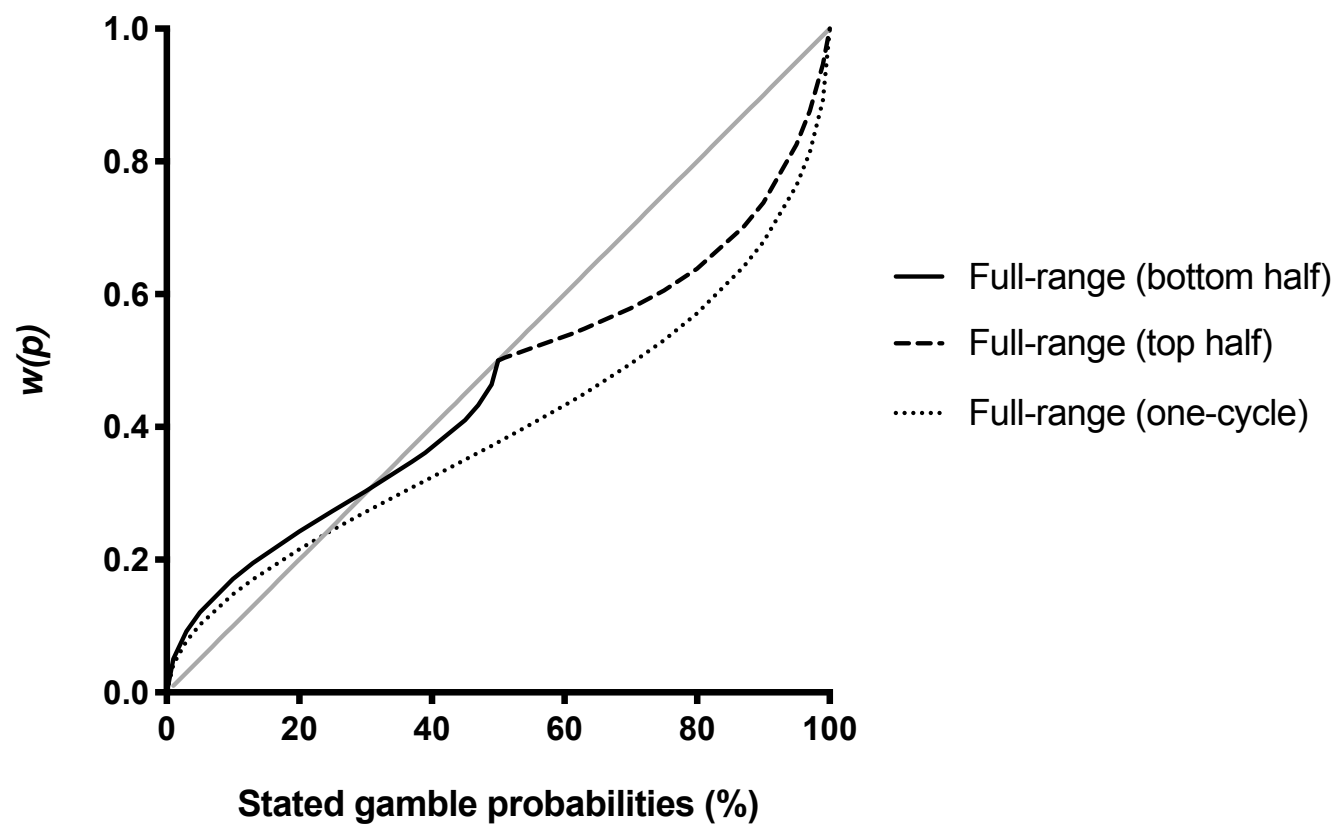




\section{Supplementary Materials}

We also fit all data using one- and two-cycle versions of a modified CPT model with Tversky and Kahneman's (1992) one-parameter probability weighting function, $w(p)=p^{\gamma} /\left(p^{\gamma}+(1-p)^{\gamma}\right)^{1 / \gamma}$ (Eq. 2 in the text) in place of the Lattimore et al. (1992) two-parameter function. We refer to this modified CPT model as CPT-TK to differentiate it from the model in the text. The modified CPT-TK equation we used for model fitting was specified as follows, where $0 \leq p \leq 1$ :

$$
\begin{aligned}
& C E=\left(x _ { 1 } ^ { \alpha } \cdot \left(\left(\left(R_{n}-R_{0}\right) /\left(R_{i}-R_{i-1}\right) \cdot\left(p-R_{i-1}\right)\right)^{\gamma} /\left(\left(\left(R_{n}-R_{0}\right) /\left(R_{i}-R_{i-1}\right) \cdot\left(p-R_{i-1}\right)\right)^{\gamma}+\left(\left(R_{n}-R_{0}\right) /\left(R_{i}-R_{i-}\right.\right.\right.\right.\right. \\
& \left.\left.\left.\left.{ }_{1}\right) \cdot\left(R_{i}-p\right)\right)^{\gamma}\right)^{1 / \gamma} \cdot\left(R_{i}-R_{i-1}\right) /\left(R_{n}-R_{0}\right)+R_{i-1}\right)+x_{2}^{\alpha} \cdot\left(1-\left(\left(\left(R_{n}-R_{0}\right) /\left(R_{i}-R_{i-1}\right) \bullet\left(p-R_{i-1}\right)\right)^{\gamma} /\left(\left(\left(R_{n}-\right.\right.\right.\right.\right. \\
& \left.\left.\left.\left.\left.\left.R_{0}\right) /\left(R_{i}-R_{i-1}\right) \bullet\left(p-R_{i-1}\right)\right)^{\gamma}+\left(\left(R_{n}-R_{0}\right) /\left(R_{i}-R_{i-1}\right) \cdot\left(R_{i}-p\right)\right)^{\gamma}\right)^{1 / \gamma} \cdot\left(R_{i}-R_{i-1}\right) /\left(R_{n}-R_{0}\right)+R_{i-1}\right)\right)\right)^{1 / \alpha}
\end{aligned}
$$

This equation is similar to Equation 11 in the text with the following notable changes: (1) there are no $\delta \mathrm{s}$, and elevation of the probability weighting curve is obtained by instead raising each probability weighting expression to $1 / \gamma ;(2) p$ and $R$ values must be input on a 0 to 1 scale to accommodate the use of the $1 / \gamma$. In the present context, this means that reference values are 0 and 1 for the one-cycle model, and $0, .5$, and 1 for the two-cycle model, and that gamble percentages are input as values between 0 and 1 (e.g., $10 \%$ is $p=.10$ ).

Primary model fit measures and deviation correlations with numeracy are provided in the tables. The one-cycle model provided a better fit than the two-cycle model in more comparisons here than in the primary report (when the Lattimore et al. probability weighting function was used). We attribute this difference to the present model being less flexible than the Lattimore et al. 
model (e.g., less able to accommodate a high crossover of the identity line). Otherwise, the conclusions from the primary text remain the same with use of this model, including that there is some evidence of a two-cycle pattern, especially in the upper-range condition, but that this pattern is not necessarily due to the manipulation of probability range by condition. 
Supplementary Table 1. Median parameter estimates and model fit measures for one-cycle versus two-cycle CPT-TK model fit to individual-level data in each condition

\begin{tabular}{lccccc}
\hline Condition & Model & $\begin{array}{c}\text { Percent } \\
\text { best fit by } \\
\text { model }\end{array}$ & $\begin{array}{c}\text { Value } \\
\text { curvature } \\
(\alpha)\end{array}$ & $\begin{array}{c}\text { Probability } \\
\text { weighting } \\
\text { curvature } \\
(\gamma)\end{array}$ & RMSE \\
\hline $\begin{array}{l}\text { Full- } \\
\text { range } \\
(n=40)\end{array}$ & one-cycle & $73 \%$ & $.95(63 \%)$ & $.51(90 \%)$ & $28.34(.91)$ \\
\hline $\begin{array}{l}\text { Upper- } \\
\text { range } \\
(n=42)\end{array}$ & one-cycle & $36 \%$ & $.95(60 \%)$ & $.53(100 \%)$ & $29.09(.91)$ \\
\hline $\begin{array}{l}\text { Lower- } \\
\text { range } \\
(n=45)\end{array}$ & one-cycle & $76 \%$ & $.82(71 \%)$ & $.62(84 \%)$ & $20.61(.89)$ \\
\hline
\end{tabular}

Notes: Data from all participants within a condition were used to produce descriptive statistics (not just one's best fit by each model). In parentheses with each parameter estimate is the percentage of participants with estimate $<1$. In parentheses with $R M S E$ is $R^{2}$ value. Two-cycle model fit reliably better than one-cycle model in upper-range condition, based on a comparison of $R M S E$ within-subjects (i.e., more participants had data better fit by the two-cycle than by the one-cycle model, even though the median RMSE is slightly lower for the latter), while the reverse was true in lower-range condition $(p \mathrm{~s}<.05)$. 
Supplementary Table 2. Median parameter estimates and model fit measures for one-cycle versus two-cycle CPT-TK model fit to individual-level data for top (50-100\%) versus bottom (0$50 \%$ ) halves of probabilities of full-range condition

\begin{tabular}{lccccc}
\hline $\begin{array}{l}\text { Full-range } \\
\text { condition } \\
\text { subset }\end{array}$ & $\begin{array}{c}\text { Percent } \\
\text { best fit by } \\
\text { model }\end{array}$ & Model & $\begin{array}{c}\text { Value } \\
\text { curvature } \\
(\alpha)\end{array}$ & $\begin{array}{c}\text { Probability } \\
\text { weighting } \\
\text { curvature } \\
(\gamma)\end{array}$ & $R M S E$ \\
\hline $50-100 \%$ & $58 \%$ & one-cycle & $1.01(45 \%)$ & $.48(90 \%)$ & $29.55(.91)$ \\
\hline $0-50 \%$ & $42 \%$ & two-cycle & $.66(78 \%)$ & $.54(75 \%)$ & $31.33(.92)$ \\
\hline & $63 \%$ & one-cycle & $.92(78 \%)$ & $.70(75 \%)$ & $19.28(.80)$ \\
\hline
\end{tabular}

$n=40$. Note: One-cycle model fit reliably better than two-cycle model for the bottom half of the data $(p<.05)$, but there was no difference in fit for the top half $(p>.100)$. 
Supplementary Table 3. Spearman correlations between deviation scores and numeracy measures

\begin{tabular}{lccc}
\hline & $\begin{array}{c}\text { Value } \\
\text { curvature } \\
\text { deviation } \\
\left(\alpha_{\mathrm{d}}\right)\end{array}$ & $\begin{array}{c}\text { Probability } \\
\text { curvature } \\
\text { deviation } \\
\left(\gamma_{\mathrm{d}}\right)\end{array}$ & $R^{2}$ \\
\hline Weller et al. numeracy & $-.18 * /-.26 * *$ & $-.14 /-.11$ & $.32 * * * / .32 * * *$ \\
Lipkus et al. numeracy & $-.20 * /-.23 * *$ & $-.02 /-.12$ & $.21 * / .20^{*}$ \\
\hline
\end{tabular}

$N=126 .{ }^{* * *} p<.001 ;{ }^{* *} p<.01 ;{ }^{*} p<.05$. Notes: First value in cell is correlation between numeracy measure and deviation score for best fitting model (one-cycle or two-cycle); second score assumes one-cycle model. 